\title{
Non-thermal radio supernova remnants of exiled Wolf-Rayet stars
}

\author{
D. M.-A. Meyer ${ }^{\oplus},{ }^{1 \star}$ M. Pohl, ${ }^{1,2}$ M. Petrov ${ }^{3}$ and L. Oskinova ${ }^{1,4}$ \\ ${ }^{1}$ Institut für Physik und Astronomie, Universität Potsdam, Karl-Liebknecht-Strasse 24/25, D-14476 Potsdam, Germany \\ ${ }^{2}$ DESY Platanenallee 6, D-15738 Zeuthen, Germany \\ ${ }^{3}$ Max Planck Computing and Data Facility (MPCDF), Gießenbachstrasse 2, D-85748 Garching, Germany \\ ${ }^{4}$ Department of Astronomy, Kazan Federal University, Kremlevskaya Str 18, Kazan, Russia
}

Accepted 2021 February 10. Received 2021 February 10; in original form 2020 December 15

\begin{abstract}
A signification fraction of Galactic massive stars $\left(\geq 8 \mathrm{M}_{\odot}\right)$ are ejected from their parent cluster and supersonically sail away through the interstellar medium (ISM). The winds of these fast-moving stars blow asymmetric bubbles thus creating a circumstellar environment in which stars eventually die with a supernova explosion. The morphology of the resulting remnant is largely governed by the circumstellar medium of the defunct progenitor star. In this paper, we present 2D magnetohydrodynamical simulations investigating the effect of the ISM magnetic field on the shape of the supernova remnants of a $35 \mathrm{M}_{\odot}$ star evolving through a Wolf-Rayet phase and running with velocity 20 and $40 \mathrm{~km} \mathrm{~s}^{-1}$, respectively. A $7 \mu \mathrm{G}$ ambient magnetic field is sufficient to modify the properties of the expanding supernova shock front and in particular to prevent the formation of filamentary structures. Prior to the supernova explosion, the compressed magnetic field in the circumstellar medium stabilizes the wind/ISM contact discontinuity in the tail of the wind bubble. A consequence is a reduced mixing efficiency of ejecta and wind materials in the inner region of the remnant, where the supernova shock wave propagates. Radiative transfer calculations for synchrotron emission reveal that the non-thermal radio emission has characteristic features reflecting the asymmetry of exiled core-collapse supernova remnants from Wolf-Rayet progenitors. Our models are qualitatively consistent with the radio appearance of several remnants of high-mass progenitors, namely the bilateral G296.5+10.0 and the shell-type remnants CTB109 and Kes 17, respectively.
\end{abstract}

Key words: radiation mechanisms: non-thermal-stars: massive-ISM: supernova remnants.

\section{INTRODUCTION}

Massive stars are born with masses $M_{\star} \geq 8 \mathrm{M}_{\odot}$. Despite their rareness, they are of prime importance in the cycle of matter in the interstellar medium (ISM) of our Galaxy (Langer 2012). After a relatively long hydrogen-burning main-sequence phase, they experience a series of evolutionary phases characterized by abrupt changes in their surface properties (radius, effective temperature, mass-loss rate, and wind velocity). Those evolutionary phases alternate between hot, possibly eruptive phases of dilute supersonic winds (Brott et al. 2011) and colder periods of inflated radius with a dense, slow stellar wind (Ekström et al. 2012). The number and characteristics of the post-main-sequence phases are, amongst other, a function of the initial mass, the intrinsic rotation (Yoon \& Langer 2005), and the chemical composition of the star (Sanyal et al. 2017). These various winds produce shells in the circumstellar medium that develop instabilities and eventually collide together (Garcia-Segura, Mac Low \& Langer 1996; Freyer, Hensler \& Yorke 2003, 2006). They chemically enrich the ISM and drive turbulence in it, on account of the large amount of momentum and energy the winds deposit in the stellar surroundings. Finally, the majority of massive stars end their life as core-collapse supernova, whose shock wave expands into their

^E-mail: dmameyer.astro@gmail.com circumstellar medium (Woosley, Heger \& Weaver 2002), shaped by stellar winds and radiation.

Wolf-Rayet stars are an advance evolutionary stage of stars with initial mass $\geq 20 \mathrm{M}_{\odot}$, generally following a supergiant phase. Their stellar winds are fast, dense, and chemically enriched in C, N, and $\mathrm{O}$ elements (Hamann, Gräfener \& Liermann 2006; Sander, Hamann \& Todt 2012; Bestenlehner et al. 2014). The interaction of fast Wolf-Rayet wind with slower wind material expelled at previous evolutionary stage results in complex stellar surroundings such as ring nebulae (Stock \& Barlow 2010; Gvaramadze, Kniazev \& Fabrika 2010a; Meyer et al. 2020) or bipolar bubbles (Gvaramadze, Kniazev \& Fabrika 2010b). A fraction of Wolf-Rayet star is ocated at high Galactic latitude (Moffat et al. 1998; Munoz et al. 2017; Toalá et al. 2018). These fast-moving Wolf-Rayet stars that left their parent stellar clusters and reached low-density regions of the ISM. There, they eventually explode as a core-collapse supernova inside the cavity carved by the stellar wind (Franco et al. 1991; Rozyczka et al. 1993; Dwarkadas 2007). Wolf-Rayet-evolving stars are therefore ideal progenitor candidates for core-collapse supernova remnants (Katsuda et al. 2018).

Several mechanisms determine the morphology of the supernova remnants of massive progenitors. Clumpiness affecting the shockwave propagation can arise from wind-wind interaction as observed in the supernova remnant Cas A (van Veelen et al. 2009). In addition to instabilities directly developing in the supernova 
explosion itself (Janka, Melson \& Summa 2016), asymmetries in supernova remnants may be a direct consequence of interactions between the expanding shock wave and an anisotropic circumstellar medium. Of prime importance for the shaping of supernova remnants is the peculiar motion of very high-mass progenitors moving through the ISM (Meyer, Petrov \& Pohl 2020). As an example, RWC 86 (Broersen et al. 2014; Gvaramadze et al. 2017) or the Cygnus Loop (Aschenbach \& Leahy 1999; Fang, Yu \& Zhang 2017) reveal features consistent with the typical characteristics of off-centre explosions in massive stellar wind bubbles, suggesting that they might have been produced by a fast-moving progenitor, see also Toledo-Roy et al. (2014). All the numerous mechanisms, which induce deviations from sphericity in supernova shock waves, can operate in parallel, providing a huge parameter space governing the evolution of core-collapse supernova remnants. Explanations of their observed morphologies are subject to degeneracies and alternative scenarios. Runaway Wolf-Rayet stars constitute therefore the ideal candidates for the production of isolated, asymmetric core-collapse supernova remnants (Meyer et al. 2015).

The structure and properties of the ISM are also involved in the shaping of supernova remnants (Ferreira \& de Jager 2008). The ISM has an intrinsic filamentary, turbulent, and magnetized nature. Its gravito-turbulent evolution, powered by the formation of massive pre-stellar cores, stellar wind outflows, and supernova feedback enriching the ISM, drives turbulence in it and participates in the formation of the next generation of stars. Native ISM magnetic field is an important player in the evolution of the circumstellar medium around massive stars. As an example, the internal physics of bow shock nebulae around runaway stars (Gvaramadze et al. 2014; Meyer et al. 2014; van Marle, Decin \& Meliani 2014; Meyer et al. 2017), as well as the organization of supernova remnants (Orlando et al. 2007; Ferreira \& de Jager 2008; Orlando et al. 2008; Schneiter et al. 2010; Orlando et al. 2012) are partially determined by the local ambient magnetic field. Furthermore, the local direction of magnetic field makes thermal conduction anisotropic (Balsara, Tilley \& Howk 2008; Meyer et al. 2017) and can suppress (magneto)hydrodynamical instabilities (Viallet \& Baty 2007; van Marle et al. 2014). Importantly, it has been shown that the magnetization of the ISM strongly elongates stellar wind bubbles around static massive stars along the direction of the local field lines (van Marle, Meliani $\&$ Marcowith 2015). The question is therefore how important for the shaping of the supernova remnants are the effects of the ISM magnetization as compared to those of the motion of runaway WolfRayet progenitors?

In this work, we investigate, by means of numerical magnetohydrodynamical (MHD) simulations, the effects of a background ISM magnetic field on the morphological evolution of supernova remnants generated by runaway massive progenitors. We adopt the standard two-dimensional axisymmetric approach developed by many authors (Comerón \& Kaper 1998; Mackey et al. 2012; Meyer et al. 2016). It consists of first modelling the pre-supernova circumstellar medium of massive progenitors before launching a supernova blastwave in it (Velázquez et al. 2006; Chiotellis, Schure \& Vink 2012; van Marle, Meliani \& Marcowith 2012; Meyer et al. 2015). We examine the remnant morphologies and perform radiative transfer calculations for their non-thermal radio synchrotron emission maps. The mixing of supernova ejecta, stellar winds, and ISM material is also discussed, comparing models with and without ISM magnetic field. Last, we discuss these remnants in the context of cosmic ray acceleration before comparing them to Galactic supernova remnants from massive progenitors.
Our study is organized as follows. First, we present the numerical methods used for the MHD simulations of supernova remnants of $35 \mathrm{M}_{\odot}$ runaway massive stars in Section 2 . We describe our results for the dynamical evolution of both the stellar surroundings and the supernova remnant, together with predictive non-thermal radio synchrotron emission maps of these objects in Section 3. We analyse therein the effects of the presence of the ISM magnetic field on to the remnants evolution. Our results are further discussed in Section 4, and finally, we present our conclusions in Section 5.

\section{NUMERICAL SIMULATIONS}

This section describes the methods used to perform simulations of the circumstellar medium of a $35 \mathrm{M}_{\odot}$ massive star evolving up to the Wolf-Rayet phase and ending its life in a supernova explosion. We simulate the stellar surroundings from the zero-age phase of the progenitor to the late phase of supernova remnant evolution, varying the velocity of the star relative to the ISM and investigating the role of the ISM magnetic field. The simulations are used for further radiative transfer calculations of non-thermal radio synchrotron emission.

\subsection{Simulation method for the pre-supernova phase}

The pre-supernova circumstellar medium around the progenitor star is the wind-blown bubble generated by interaction between the stellar wind and the local ISM. We simulate it as described in Meyer et al. (2020). We first perform 2D cylindrical, axisymmetric, magnetohydrodynamics numerical models with a coordinate system $\left[z_{\min }\right.$; $\left.z_{\max }\right] \times\left[O ; R_{\max }\right]$ that is mapped with a uniform grid of spatial resolution $R_{\max } / N_{\mathrm{R}}$. The stellar wind of the $35 \mathrm{M}_{\odot}$ star is released at the centre of the domain into a uniformly distributed ISM. A circular wind zone of radius 20 cells is filled with the wind density profiles,

$\rho_{w}(r)=\frac{\dot{M}}{4 \pi r^{2} v_{\mathrm{w}}}$

where $\dot{M}$ is the wind mass-loss rate at different evolutionary phases interpolated from a stellar evolutionary track, $r$ is the distance to the origin of the domain, $O$, and $v_{\mathrm{w}}$ is the velocity of the stellar wind (Comerón \& Kaper 1998; van Marle et al. 2011, 2014).

In Fig. 1, we show the evolutionary path of the star and its wind, which we use in the simulations. The stellar mass (panel a, in $\mathrm{M}_{\odot}$ ), the mass-loss rate (panel $\mathrm{b}$, in $\mathrm{M}_{\odot} \mathrm{yr}^{-1}$ ), and the terminal wind velocity (panel c, in $\mathrm{km} \mathrm{s}^{-1}$ ) are displayed beginning at the age $3 \mathrm{Myr}$. The wind properties of this zero-age-main-sequence, non-rotating $35-\mathrm{M}_{\odot}$ star at Galactic metallicity has been interpolated from the Geneva library of stellar models calculated with the GENEC code (Ekström et al. 2012) by means of the online interface SYCLIST. ${ }^{1}$ The terminal speed, $v_{\mathrm{w}}$, is modified for high effective temperatures and massive stars using the approximation of Eldridge et al. (2006):

$v_{\mathrm{w}}=\sqrt{\beta(T)} v_{\mathrm{esc}}=\sqrt{\beta(T) \frac{2 G M_{\star}}{R_{\star}}}$,

where $v_{\text {esc }}$ is the escape speed of the star, $R_{\star}$ the stellar radius, and

$\beta_{\mathrm{w}}(T)= \begin{cases}1.0 & \text { if } T \leq 10000 \mathrm{~K}, \\ 1.4 & \text { if } T \leq 21000 \mathrm{~K}, \\ 2.65 & \text { if } T>21000 \mathrm{~K}\end{cases}$

a corrective function depending on the temperature $T$.

${ }^{1}$ https://www.unige.ch/sciences/astro/evolution/en/database/syclist/ 


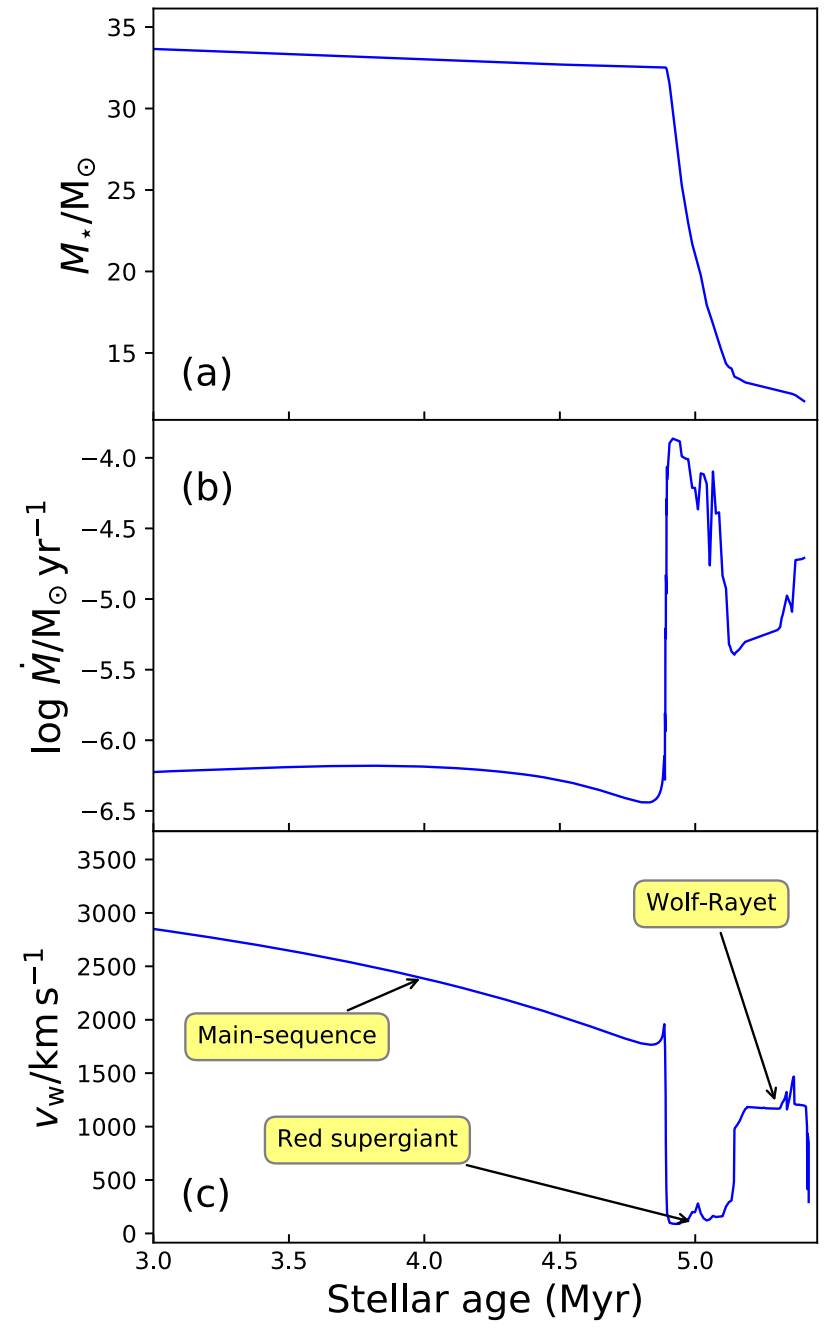

Figure 1. Stellar properties at the end of the main-sequence and during the post-main-sequence evolution of the $35 \mathrm{M}_{\odot}$ star. The panels show the stellar mass (top, panel a), mass-loss rate (middle, panel b), and wind velocity (bottom, panel c) as a function of time (in Myr).

The star first experiences a rather long main-sequence phase lasting about $4.8 \mathrm{Myr}$, blowing winds with $\dot{M} \approx 10^{-6.2} \mathrm{M}_{\odot} \mathrm{yr}^{-1}$ and $v_{\mathrm{w}} \approx 3000 \mathrm{~km} \mathrm{~s}^{-1}$. After the long main-sequence phase, the star becomes cooler and inflates to become a red supergiant with mass-loss rate $\dot{M} \approx 10^{-4} \mathrm{M}_{\odot} \mathrm{yr}^{-1}$ and wind speed $v_{\mathrm{w}} \approx 50 \mathrm{~km} \mathrm{~s}^{-1}$. It finally evolves to the Wolf-Rayet phase, characterized by both a high mass-loss rate $\left(\dot{M} \approx 10^{-5.0} \mathrm{M}_{\odot} \mathrm{yr}^{-1}\right)$ and a large wind speed $\left(v_{\mathrm{w}} \approx 1500 \mathrm{~km} \mathrm{~s}^{-1}\right)$.

To study the circumstellar medium around runaway Wolf-Rayet stars, we conducted a series of simulations with varying stellar velocities spanning from $v_{\star}=10$ to $v_{\star}=40 \mathrm{~km} \mathrm{~s}^{-1}$. The star moves in $z$-direction, and we simulate in the frame of the star, ISM gas of number density $n_{\text {ISM }} \approx 0.79 \mathrm{~cm}^{-3}$ and temperature $T_{\text {ISM }} \approx 8000 \mathrm{~K}$ as in the $\mathrm{H}$ II regions around hot stars. The ISM material flows in with speed $v_{\star}$ at the boundary $z=z_{\max }$. Outflow boundaries conditions are set at $z=z_{\min }$ and $R=R_{\max }$, respectively. Each value of $v_{\star}$ is explored with and without magnetization of the ISM. The ISM magnetic field direction is parallel to the $O z$ axis, as a direct consequence of the simulation geometry, and it is set to $B_{\mathrm{ISM}}=7 \mu \mathrm{G}$ that is typical value for the warm phase of the ISM (van Marle et al. 2014, 2015; Meyer et al. 2017). The flow of material past the stellar wind is characterized by the Alfvén speed:

$v_{\mathrm{A}}=\sqrt{\frac{\boldsymbol{B}_{\mathrm{ISM}} \cdot \boldsymbol{B}_{\mathrm{ISM}}}{4 \pi n m_{\mathrm{H}}}}$,

which together with the sound speed (equation 18) determines the Alfvénic and sonic Mach number of the stellar wind bubble in the ISM. We list both for each model in Table 1.

A continuity equation,

$\frac{\partial\left(\rho Q_{1}\right)}{\partial t}+\nabla \cdot\left(\boldsymbol{v} \rho Q_{1}\right)=0$,

is used to trace the mixing of stellar wind material into the ISM, with $\rho$ the mass density, respectively. Initially, the tracer $Q_{1}$ is set to $Q_{1}(\boldsymbol{r})=1$ in the wind and to $Q_{1}(\boldsymbol{r})=0$ in the ISM.

\subsection{Supernova explosion}

After establishing the circumstellar medium around the presupernova massive star, we simulate the supernova explosion as a spherically symmetric shock wave expanding into the freely expanding stellar wind of the progenitor. The supernova-wind interaction then serves as initial condition of a subsequent two-dimensional calculation of the corresponding remnant (Meyer et al. 2015, 2020). The properties of the blastwave are parametrized by the explosion energy, $E_{\mathrm{ej}}=10^{51} \mathrm{erg}$, and the ejecta mass,

$M_{\mathrm{ej}}=M_{\star}-\int_{t_{\mathrm{ZAMS}}}^{t_{\mathrm{SN}}} \dot{M}(t) d t-M_{\mathrm{NS}}=11.64 \mathrm{M}_{\odot}$,

where $t_{\text {ZAMS }}$ and $t_{\mathrm{SN}}$ denote the times of zero age and supernova, respectively, and $M_{\mathrm{NS}}=1.4 \mathrm{M}_{\odot}$ is the mass of the remnant neutron star left behind the supernova explosion. Note that we use the canonical explosion energy typically taken in hydrodynamical simulations of supernova remnants (van Veelen et al. 2009; van Marle et al. 2010, 2012). However, detailed dedicated studies estimate the energy released throughout the explosion of a core-collapse progenitor to be rather in the range $E_{\mathrm{ej}}=1-5 \times 10^{50} \mathrm{erg}$ (Smartt 2009; Janka 2012; Moriya et al. 2018). A passive scalar, $Q_{2}(\boldsymbol{r})$, obeying the continuity equation,

$\frac{\partial\left(\rho Q_{2}\right)}{\partial t}+\nabla \cdot\left(\boldsymbol{v} \rho Q_{2}\right)=0$,

is used to distinguish supernova ejecta from stellar wind or ISM material, by setting $Q_{2}(\boldsymbol{r})=1$ in the supernova-ejecta region and $Q_{2}(\boldsymbol{r})=0$ otherwise.

The supernova shock wave is released into the progenitor's stellar wind bubble (Whalen et al. 2008; Zirakashvili \& Ptuskin 2018) by superposing a 1D blastwave density profile, $\rho(r)$, on to the presupernova wind distribution. We used a typical ejecta profile for the early expansion of a core-collapse supernovae. It involves a homologuous expansion, $v=r / t$, the radius of the progenitor star's core at the time of the supernova, $r_{\text {core }}$, and the outermost extension $r_{\max }$ of the blastwave. We start the calculations at

$t_{\max }=\frac{r_{\max }}{v_{\max }}$,

where $v_{\max }=30000 \mathrm{~km} \mathrm{~s}^{-1}$ is the ejecta velocity at $r_{\max }$ (van Veelen et al. 2009). The value of $r_{\max }$ is determined by the explosion energy and ejecta mass (Whalen et al. 2008). The density profile of the ejecta is set as

$\rho(r)= \begin{cases}\rho_{\text {core }}(r) & \text { if } r \leq r_{\text {core }}, \\ \rho_{\text {max }}(r) & \text { if } r_{\text {core }}<r<r_{\text {max }},\end{cases}$ 
Table 1. List of models. The columns indicate the velocity of the star, $v_{\star}$, the grid resolution and size in pc, and the sonic and Alfénic Mach number of the moving star with respect to the ISM. The runs are labelled 'CSM' for the pre-supernova modelling and 'SNR' for the remnant simulations, and likewise 'HD' for hydrodynamics and 'MHD' for magneto-hydrodynamics.

\begin{tabular}{lccccc}
\hline Model & $v_{\star}\left(\mathrm{km} \mathrm{s}^{-1}\right)$ & Grid size & Grid mesh & $M$ & $M_{\mathrm{A}}$ \\
\hline Run-35-MHD-20-CSM & 20 & {$[0 ; 175] \times[-250 ; 100]$} & $2000 \times 4000$ cells & 1.0 & 1.16 \\
Run-35-HD-20-CSM & 20 & {$[0 ; 175] \times[-250 ; 100]$} & $2000 \times 4000$ cells & 1.0 & 1.16 \\
Run-35-MHD-40-CSM & 40 & {$[0 ; 150] \times[-300 ; 100]$} & $1500 \times 4000$ cells & 2.0 & 2.32 \\
Run-35-HD-40-CSM & 40 & {$[0 ; 150] \times[-300 ; 100]$} & $1500 \times 4000$ cells & 2.0 & 2.32 \\
Run-35-MHD-20-SNR & 20 & {$[0 ; 200] \times[-275 ; 175]$} & $4000 \times 9000$ cells & 1.0 & 1.16 \\
Run-35-HD-20-SNR & 20 & {$[0 ; 200] \times[-275 ; 175]$} & $4000 \times 9000$ cells & 1.0 & 1.16 \\
Run-35-MHD-40-SNR & 40 & {$[0 ; 200] \times[-330 ; 170]$} & $4000 \times 10000$ cells & 2.0 & 2.32 \\
Run-35-HD-40-SNR & 40 & {$[0 ; 200] \times[-330 ; 170]$} & $4000 \times 10000$ cells & 2.0 & 2.32 \\
\hline
\end{tabular}

where

$\rho_{\text {core }}(r)=\frac{1}{4 \pi n} \frac{\left(10 E_{\mathrm{ej}}^{n-5}\right)^{-3 / 2}}{\left(3 M_{\mathrm{ej}}^{n-3}\right)^{-5 / 2}} \frac{1}{t_{\max }^{3}}$,

is constant, whereas the ejecta density further out follows a power law,

$\rho_{\max }(r)=\frac{1}{4 \pi n} \frac{\left(10 E_{\mathrm{ej}}^{n-5}\right)^{(n-3) / 2}}{\left(3 M_{\mathrm{ej}}^{n-3}\right)^{(n-5) / 2}}\left(\frac{r}{t_{\max }}\right)^{-n}$,

with $n=11$ (Chevalier 1982; Truelove \& McKee 1999).

Beyond $r_{\max }$, the density profile is that of the freely expanding wind as found in the pre-supernova wind bubble simulations. The ejecta speed at the distance $r_{\text {core }}$ from the centre of the explosion is (Truelove \& McKee 1999)

$v_{\text {core }}=\left(\frac{10(n-5) E_{\mathrm{ej}}}{3(n-3) M_{\mathrm{ej}}}\right)^{1 / 2}$.

This 1D ejecta-wind interaction solution is mapped on to the 2D domain of the subsequent simulation. We integrate the equations up to $150 \mathrm{kyr}$ after the supernova.

\subsection{Governing equations}

The dynamics of a magnetized flow is described by the equations of ideal magneto-hydrodynamics plus losses and heating by optically thin radiation,

$$
\begin{aligned}
& \frac{\partial \rho}{\partial t}+\nabla \cdot(\rho \boldsymbol{v})=0, \\
& \frac{\partial \boldsymbol{m}}{\partial t}+\nabla \cdot\left(\boldsymbol{m} \otimes \boldsymbol{v}+\boldsymbol{B} \otimes \boldsymbol{B}+\hat{\mathbf{I}} p_{\mathrm{t}}\right)=\mathbf{0}, \\
& \frac{\partial E}{\partial t}+\nabla \cdot\left(\left(E+p_{\mathrm{t}}\right) \boldsymbol{v}-\boldsymbol{B}(\boldsymbol{v} \cdot \boldsymbol{B})\right)=\Phi(T, \rho),
\end{aligned}
$$

and

$\frac{\partial \boldsymbol{B}}{\partial t}+\nabla \cdot(\boldsymbol{v} \otimes \boldsymbol{B}-\boldsymbol{B} \otimes \boldsymbol{v})=\mathbf{0}$,

with the linear momentum vector, $\boldsymbol{m}=\rho \boldsymbol{v}$, and the magnetic field vector, $\boldsymbol{B}$. The total energy of the system reads

$E=\frac{p}{(\gamma-1)}+\frac{\boldsymbol{m} \cdot \boldsymbol{m}}{2 \rho}+\frac{\boldsymbol{B} \cdot \boldsymbol{B}}{2}$,

where $\gamma=5 / 3$ is the adiabatic index for ideal gas and $p$ is the thermal pressure. The definition of the adiabatic sound speed,

$c_{\mathrm{s}}=\sqrt{\frac{\gamma p}{\rho}}$, closes the system, which we integrate using the so-called eight-wave algorithm. This second-order unsplit scheme satisfies $\boldsymbol{\nabla} \cdot \boldsymbol{B}=\mathbf{0}$. The time-march of the algorithm obeys the standard Courant-FriedrichLevy condition that is set to $C_{\mathrm{cfl}}=0.1$ at the beginning of the simulations.

The source term,

$\Phi(T, \rho)=n_{\mathrm{H}} \Gamma(T)-n_{\mathrm{H}}^{2} \Lambda(T)$,

accounts for optically thin radiative cooling, $\Lambda(T)$, and heating, $\Gamma(T)$. The gas temperature is

$T=\mu \frac{m_{\mathrm{H}}}{k_{\mathrm{B}}} \frac{p}{\rho}$,

where $\mu=0.61$ is the mean molecular weight, $k_{\mathrm{B}}$ the Boltzmann constant, and $m_{\mathrm{H}}$ the proton mass. The hydrogen number density is computed as

$n_{\mathrm{H}}=\frac{\rho}{\mu\left(1+\chi_{\mathrm{He}, \mathrm{Z}}\right) m_{\mathrm{H}}}$,

with $\chi_{\mathrm{He}, \mathrm{Z}}$ the mass fraction of all coolants heavier than $\mathrm{H}$. The functions $\Gamma(T)$ and $\Lambda(T)$ are described in details in Meyer et al. (2017).

\subsection{Non-thermal synchrotron radio emission}

The forward shock of the supernova remnant in particular will accelerate charged particles, such as electrons, to high energy. In the presence of magnetic field relativistic electrons produce synchrotron emission that is an excellent diagnostic (Reynolds 2008). To permit a comparison with the vast observational data of non-thermal radio synchrotron emission from supernova remnants, we produce synthetic emission maps on the basis of our MHD simulations.

Energy losses of GeV-scale electrons are likely negligible, and so we assume the electron spectrum,

$N(E)=K E^{-s}$,

where $E$ denotes the electron energy and the index, $s=2$, is expected for a strong shock. Diffusive transport is typically slower than advection in the $\mathrm{GeV}$ band, and so the accelerated electron density follows the gas density and is in fact proportional to it, if the injection efficiency at the forward shock is a constant (Drury 1983a,b). Amongst the several prescriptions for the non-thermal synchrotron emission coefficient of a magnetized gas available in the literature, we choose to use that of Jun \& Norman (1996). We refer the reader interested in details regarding to our choice of emission coefficient within this core-collapse supernova remnant problem in our Appendix A. Therefore, at a given frequency, $v$, the 
radio synchrotron emission coefficient reads

$j_{\text {sync }}(v) \propto K^{2-s} p^{s-1} B_{\perp}^{(s+1) / 2} v^{-(s-1) / 2}$,

which reduces to

$j_{\text {sync }}(v) \propto n^{2-s} p^{s-1} B_{\perp}^{(s+1) / 2} v^{-(s-1) / 2}$,

where $p$ is the gas thermal pressure and $B_{\perp}$ is the magnetic-field component perpendicular to the line of sight.

Let $\vec{l}$ be the unit vector of the observer's line of sight. Defining the viewing angle of the observer as $\theta_{\text {obs }}=\angle(\vec{l}, \vec{B})$, the total strength of the magnetic field and its perpendicular component are obtained as

$B_{\perp}=|\vec{B}| \sin \left(\theta_{\text {obs }}\right)$

and

$|\vec{B}|=\sqrt{B_{\mathrm{R}}^{2}+B_{\mathrm{z}}^{2}}$.

Then, at a given frequency the emission coefficient finally reads

$j_{\text {sync }}\left(\theta_{\text {obs }}\right) \propto n^{2-s} p^{s-1}\left(|\vec{B}| \sqrt{1-\left(\frac{\vec{B} \cdot \vec{l}}{|\vec{B}|}\right)^{2}}\right)^{(s+1) / 2}$,

which we use in our radiative transfer calculations.

For each simulation, we selected snapshots that are representative of the phases of the supernova remnant evolution, namely at times 6 , 20 , and $40 \mathrm{kyr}$ after the explosion, respectively. The corresponding density, temperature, and magnetic-field distributions are first translated from the two-dimensional cylindrical coordinates system to a three-dimensional spherical coordinate system $(r, \theta, \phi)$ with $512^{3}$ cells and the same origin, for which we rotate the cylindrical solution around the symmetry axis. On each grid zone, we pre-calculate the local component of the magnetic field that is normal to line of sight of the observer. Finally, we use a modified version of the radiative transfer code RADMC- $3 \mathrm{D}^{2}$ to perform ray-tracing integration of the radio synchrotron emission coefficient along a given line of sight with aspect angle $\theta_{\mathrm{obs}}$. The non-thermal radio intensity,

$I=\int_{\mathrm{SNR}} j_{\mathrm{sync}}\left(\theta_{\mathrm{obs}}\right) \mathrm{d} l$,

is then used to synthesize normalized emission maps.

\section{RESULTS}

We present in this section the results for the pre- and post-supernova circumstellar medium of a runaway Galactic $35 \mathrm{M}_{\odot}$ progenitor star, investigate how the stellar motion and the magnetization of the ISM affect the mixing of materials, and present the evolution of their projected radio synchrotron emission.

\subsection{Pre-supernova circumstellar medium of runaway stars}

Fig. 2 presents our results for a star moving with $v_{\star}=20 \mathrm{~km} \mathrm{~s}^{-1}$, comparing the hydrodynamical picture (top) with the MHD description (bottom). The red isocontours trace $Q_{1}=0.5$, which marks the places in the remnant with a 50/50 proportion of ISM and stellar wind. The wind of the massive star generated an ovoid bubble of size $\sim 100$ pc (Fig. 2a) in which the star is off-centred on account of stellar motion (cf. Weaver et al. 1977; Meyer et al. 2020). The large-scale stellar-wind bow shock is organized according to the classical picture of Weaver et al. (1977), made of an inner termination

${ }^{2}$ https://www.ita.uni-heidelberg.de/ dullemond/software/radmc-3d/ shock, a contact discontinuity, and an outer forward shock. They distinguish the expanding stellar wind, the hot low-density shocked wind, the cold dense ISM gas, and the ambient medium, respectively. The post-main-sequence wind, i.e. the red supergiant and WolfRayet materials, are released inside expanding stellar wind and develop instabilities at the interface separating the cold and hot gas (Fig. 2).

For a larger speed of the star, $v_{\star}=40 \mathrm{~km} \mathrm{~s}^{-1}$, the spherical symmetry is broken, and the star reaches the forward shock of its own wind bubble, itself distorted under the effects of stellar motion (Fig. 3a,b). A chimney of unperturbed stellar wind is carved into the layer of shocked ISM, and the post-main-sequence wind is blown through the tube (red isocontours). This phenomenon is even more pronounced in the case of a very fast progenitor star with $v_{\star}=70 \mathrm{~km} \mathrm{~s}^{-1}$ (Meyer et al. 2015). Interestingly, once the star has left its wind bubble, direct wind-ISM interaction resumes at the distance

$R_{\mathrm{SO}}=\sqrt{\frac{\dot{M} v_{\mathrm{w}}}{4 \pi n_{\mathrm{ISM}} v_{\star}^{2}}}$,

and a new bow shock forms (Baranov, Krasnobaev \& Kulikovskii 1971). An analytic estimate for the location of the contact discontinuity of bow shocks reads

$\frac{R(\theta)}{R_{\mathrm{SO}}}=\frac{\sqrt{3(1-\theta) \operatorname{cotan}(\theta)}}{\sin (\theta)}$,

where $\theta$ is the angle to the direction of stellar motion (Wilkin 1996). This bow shock is in its turn subject to instabilities (Brighenti \& D'Ercole 1995a,b) and constitutes the location in which the supernova explosion takes place (Brighenti \& D'Ercole 1994; Chiotellis et al. 2012; Meyer et al. 2015).

The interstellar magnetic field has strong impact on the distribution of shocked ISM, as demonstrated in van Marle et al. (2015). As an example, in Figs 2(b) and 3(b) one clearly sees that the layer of shocked ISM is puffed up along the local magnetic field as a result of the damping of Alfvén waves (van Marle et al. 2015). The effect is weaker for a fast-moving progenitor on account of the lower ratio of magnetic and ram pressure (Fig. 3b). The entire interior structure of the wind bubble is elongated when the star moves quickly. The magnetic field lines are aligned with the termination shock and discontinuities, providing additional pressure that modifies the circumstellar gas dynamics (Figs 2b). It can also damp instabilities at the contact discontinuity between hot shocked wind and cold shocked ISM gas, primarily in the tail of the wind bubble. Our models combine the asymmetry in the stellar wind bubbles of moving stars (Meyer et al. 2015) with the magnetic-pressure effect that were previously explored for static stars (van Marle et al. 2015).

\subsection{Supernova remnants}

Fig. 4 displays the structure of the supernova remnant at time $40 \mathrm{kyr}$ after the explosion. Each panel corresponds to a different simulation, with density plotted on the left-hand part of the panel and the temperature on the right-hand part of the panel, respectively. The blue isocontour marks the regions with 10 percent abundance of ejecta in number density. The left-hand panels are derived from hydrodynamical simulations, to be compared with MHD results on the right. The stellar velocities are $v_{\star}=20 \mathrm{~km} \mathrm{~s}^{-1}$ (top) and $v_{\star}=40 \mathrm{~km} \mathrm{~s}^{-1}$ (bottom), respectively.

The shape of the supernova remnants is governed by the distribution of the pre-supernova circumstellar medium (Meyer et al. 2015, 

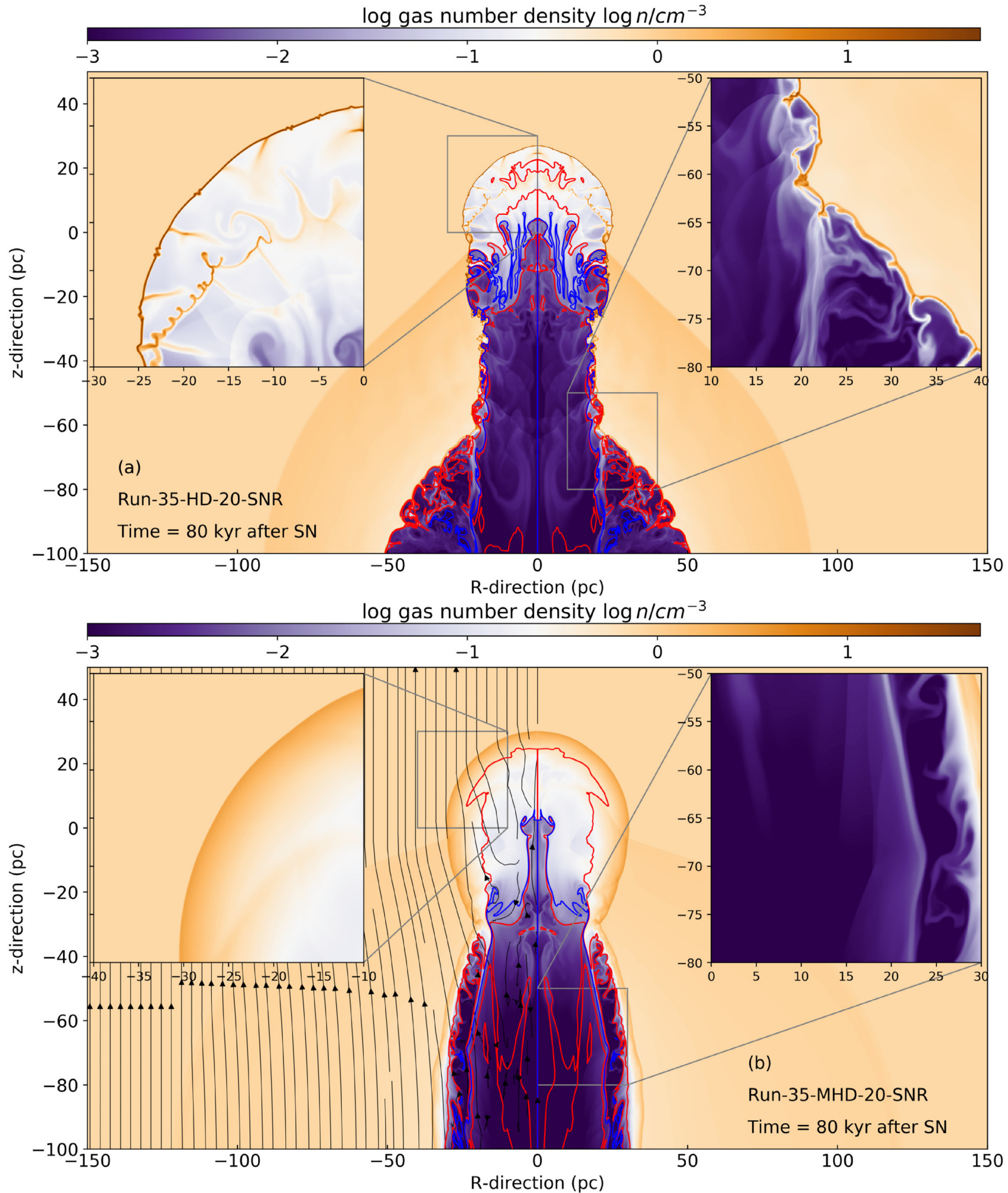

Figure 2. Density rendering of the supernova remnant of a $35-\mathrm{M}_{\odot}$ progenitor moving with $v_{\star}=20 \mathrm{~km} \mathrm{~s}^{-1}$ through uniform ISM of number density $n_{\mathrm{ISM}}=$ $0.78 \mathrm{~cm}^{-3}$. Before exploding, the remnant passed through main-sequence, red supergiant, and Wolf-Rayet phases (Ekström et al. 2012). The top panel displays the hydrodynamical model, whereas the bottom one shows the magneto-hydrodynamical picture for a 7- $\mu \mathrm{G}$ ambient magnetic field oriented parallel to the stellar motion in $z$-direction. Inset boxes highlight the dynamic filamentary structures developing from ejecta-wind-ISM interactions (left inset) and the structure of the stellar wind cavity produced by the progenitor's motion and located behind the centre of the explosion (right inset). The red lines are iso-temperature contours $\left(T=10^{6}\right.$ and $\left.10^{7} \mathrm{~K}\right)$, and the blue contours trace the region with a 10 per cent contribution of supernova ejecta in number density. 


\section{log gas number density $\log \mathrm{n} / \mathrm{cm}^{-3}$}
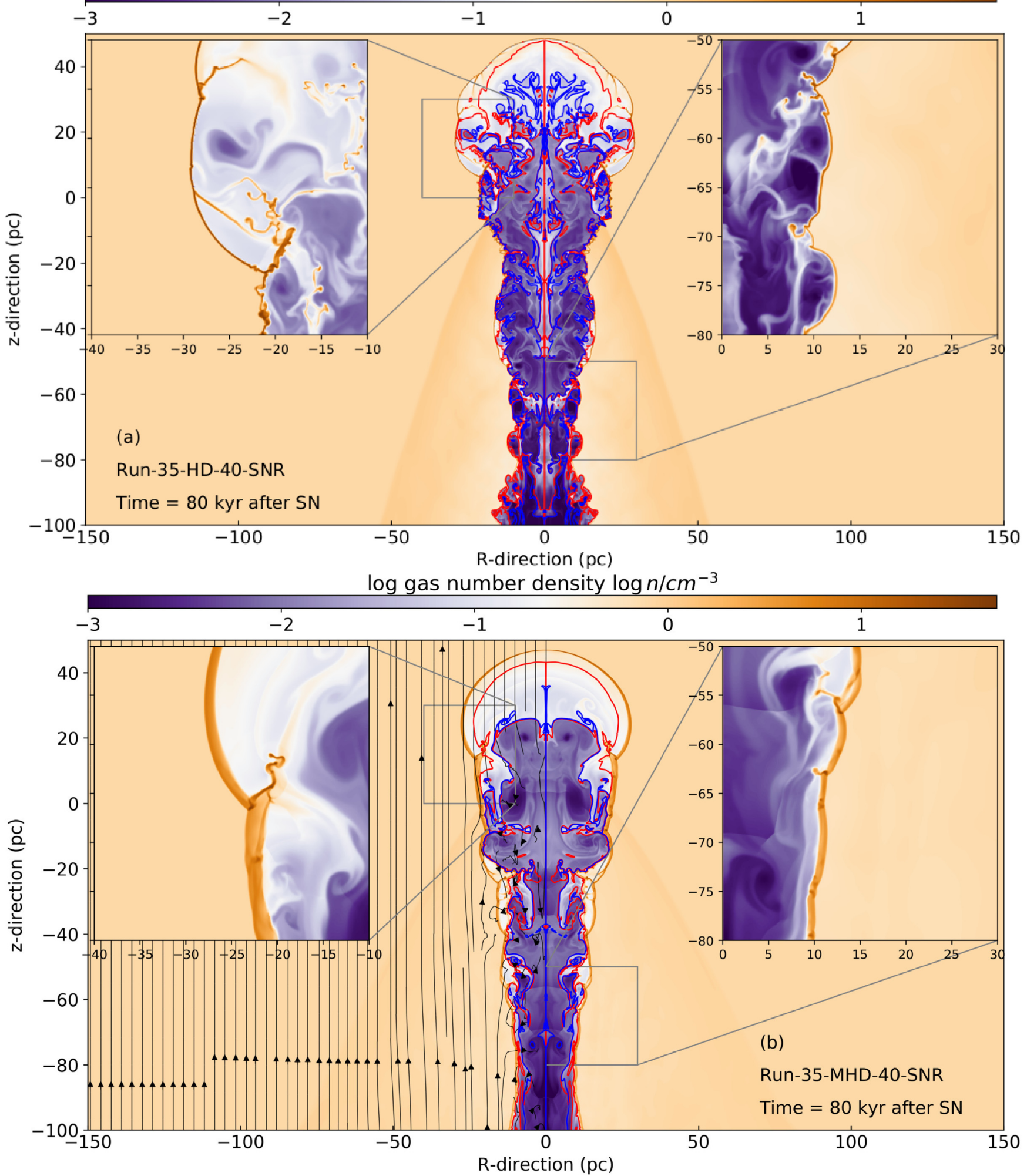

Figure 3. As Fig. 2, but for the supernova remnant of a progenitor moving with $40 \mathrm{~km} \mathrm{~s}^{-1}$.

2020). Fig. 4(b,d) show that the faster the progenitor moves through the ISM, the sooner the supernova shock wave interacts with the termination shock of the progenitor's wind bubble. For smaller $v_{\star}$, the elongated shape of the MHD wind bubble permits the WolfRayet wind to expand freely into the unperturbed red supergiant stellar wind and to generate by wind-wind collision a ring of dense swept-up material (Meyer et al. 2020), inside which the blastwave is subsequently released and expands spherically. A similar situation has been explored for static progenitor in the context of Cas A (van Veelen et al. 2009). This phenomenon is particularly prominent for 

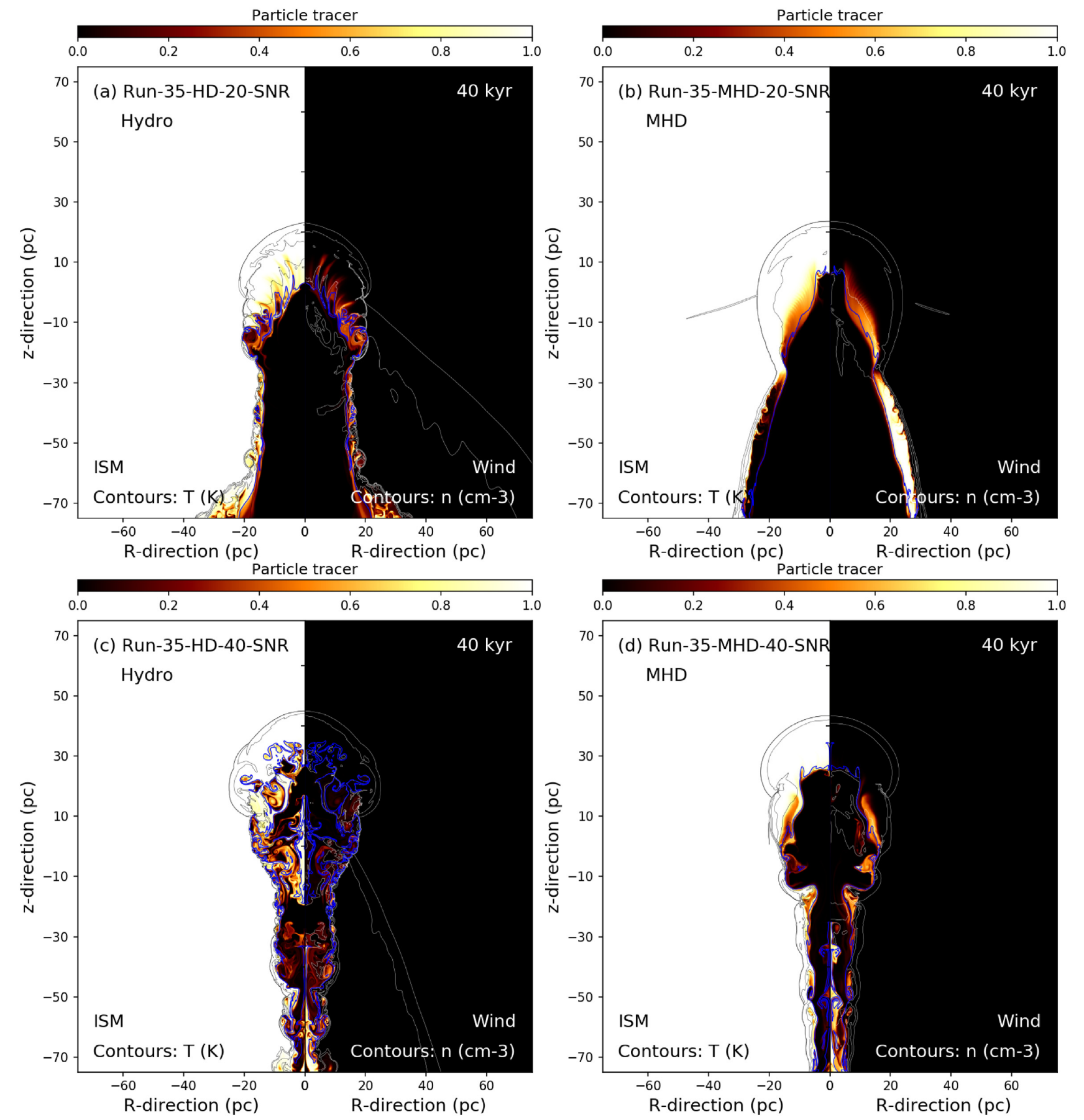

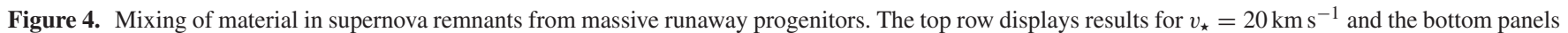

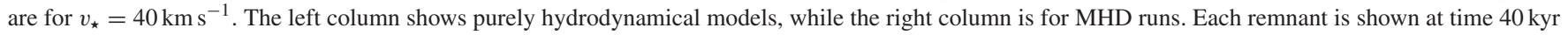

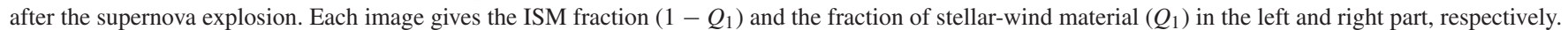

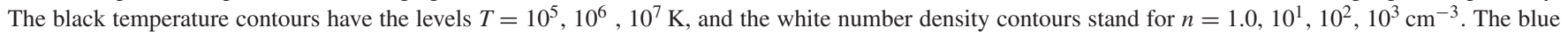
contours make the locations with 10 per cent ejecta fraction by number.

small ISM density, $n_{\text {ISM }} \ll 1$, since the radius of the main-sequence wind termination shock is much larger (van Marle et al. 2015), and so is the region filled by the last free-streaming wind.

The effects of the ISM magnetic field are also more pronounced for low progenitor speed, $v_{\star}=20 \mathrm{~km} \mathrm{~s}^{-1}$. The supernova remnant shock wave rapidly interacts with the wind bubble in the progenitor's direction of motion, and it is first reverberated towards the centre of the explosion and subsequently channelled into the wind cavity carved during the main-sequence phase, inducing a hot region hosting a lot of mixing of wind, ejecta, and shocked ISM gas (Fig. 4a). The reflections are different in the MHD case, where they occur both parallel and normal to the progenitor's motion, on account of the tubular shape of the shocked-wind region (Fig. 4b,d). A different morphology arises for fast-moving progenitors, with a rather unmixed lobe of shocked ISM ahead of the stellar motion, and a channelled region of mixed ejecta and wind material in the tail (Fig. 4c,d). 


\subsection{Effect of ISM magnetic field on the remnant's properties}

Magnetic field changes the morphology of the pre-supernova stellar wind bubble, which will eventually influence the structure of the remnant and the mixing of material in it. At a magnetized shock, the component of the magnetic field along the shock normal remains unchanged, and that in the shock plane is compressed (Shu 1992). This results in an increased magnetic pressure in the shocked ISM and, consequently, in an enlargement of the bubble perpendicular to the direction of motion, which for static wind bubbles has been demonstrated by van Marle et al. (2015). Further effects are a reduced compression ratio of the forward shock and a puffing-up of the shocked ISM gas layer. Although the MHD jump conditions imply that the ISM field is not compressed ahead of the star, since the cylindrical coordinate system imposes a parallel field, we cannot exclude that this is an artefact. We refer the reader to the thorough discussion in Meyer et al. (2017). Full three-dimensional simulations of both the pre-supernova and remnant phase of moving massive progenitor star are necessary to address this question in appropriate detail.

\subsection{Radio synchrotron emission maps}

\subsubsection{Basic consideration}

We perform a back-of-the-envelope estimate of the validity of our assumptions regarding the radio synchrotron emission. We select onedimensional cross-section of the flow variables $\rho, p$, and $T$ originating from the centre of the explosion and following the direction of stellar motion, along the axis of symmetry, $\mathrm{Oz}$, in the models, respectively. We then measure the time-dependent position, $R_{\mathrm{FS}}(t)$, and speed, $v_{\mathrm{FS}}(t)$, of the forward shock, using the shock-finder algorithm of the RATPAC code (Telezhinsky, Dwarkadas \& Pohl 2012, 2013; Bhatt et al. 2020). The radiation synthesis is based on the assumption that the forward shock accelerates a certain fraction of particles passing through it, and that the subsequent transport of radio-emitting electrons in the downstream region is entirely advective.

In our radiation transfer model, diffusion is neglected and we shall now verify this assumption. Upstream of the forward shock the density of electrons re-accelerated as cosmic rays exponentially decreases, $N(E, x) \propto e^{-x / x_{\mathrm{c}}(t)}$ with length-scale:

$x_{\mathrm{c}}(t)=\frac{D(E)}{v_{\mathrm{FS}}(t)}$,

where $D(E)$ is the diffusion coefficient and $E$ is the energy of the electrons. For simplicity, we shall assume that the diffusion coefficient is a multiple of the Bohm limit, $D(E) \approx \eta D_{\mathrm{Bohm}}(E)=\eta c / 3 r_{\mathrm{L}}(E)$, where $r_{\mathrm{L}}$ denotes the Larmor radius of the electrons and $\eta$ is a scalar controlling the . Using the characteristic frequency of synchrotron radiation, equation (31) can be rewritten as

$\frac{x_{\mathrm{c}}(t)}{\mathrm{pc}} \simeq \frac{\eta}{3000} \sqrt{\frac{v_{\mathrm{syn}}}{10 \mathrm{GHz}}}\left(\frac{v_{\mathrm{FS}}(t)}{3000 \mathrm{~km} \mathrm{~s}^{-1}}\right)^{-1}\left(\frac{B(t)}{10 \mu \mathrm{G}}\right)^{-1.5}$.

It is evident that for reasonably well-developed cosmic ray scattering ( $\eta \ll 1000)$, the precursor of radio-emitting electrons is tiny compared to the size of the system and produces a negligible contribution of synchrotron emission. Note that a significant abundance of magnetic field perpendicular the shock surface will slow down diffusive transport away from the shock and hence reduces $\eta$.

Diffusion in the downstream region may transport electrons beyond the discontinuity to the ejecta region. Within time $t$, advective transport displaces electrons from the shock by the distance $\Delta_{\mathrm{adv}} \simeq t v_{\mathrm{FS}}(t) / 4$. Diffusive transport displaces by $\Delta_{\mathrm{dif}} \simeq \sqrt{D(E) t}$,

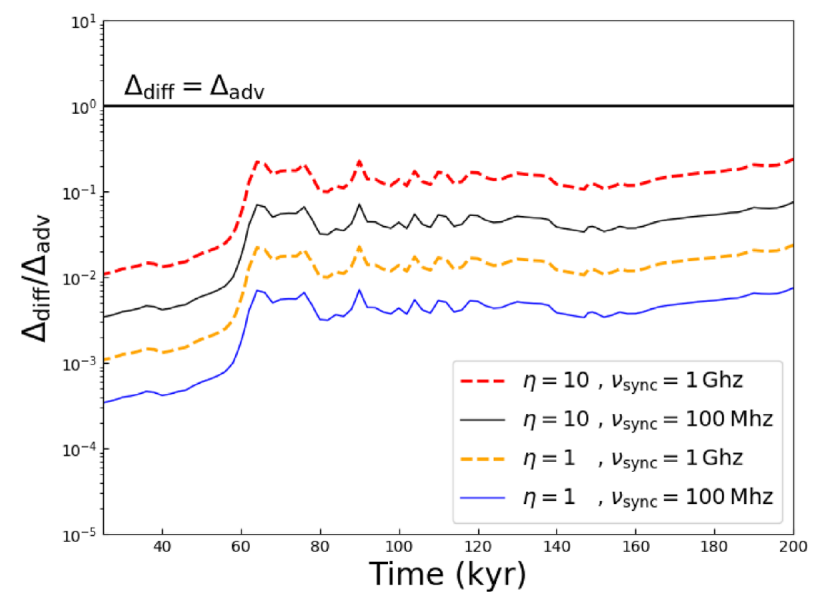

Figure 5. Comparison between the diffusion time-scale, $\Delta_{\text {diff, }}$ and the advection time-scale, $\Delta_{\mathrm{adv}}$, of a particle accelerated at the forward shock of the supernova remnant in our simulation Run-35-MHD-40-SNR of a $35-\mathrm{M}_{\odot}$ progenitor moving with $v_{\star}=40 \mathrm{~km} \mathrm{~s}^{-1}$. The quantity $\Delta_{\text {diff }} / \Delta_{\text {adv }}$ is plotted as a function of remnant age for several values of $\eta$ and synchrotron frequency $v_{\text {sync }}$, respectively. Values well below unity imply that diffusion can be neglected in the estimate of radio emission maps.

and so ignorability of diffusion requires

$t v_{\mathrm{FS}}(t) / 4 \gg \sqrt{D(E) t} \Rightarrow t v_{\mathrm{FS}}(t) \gg 16 x_{\mathrm{c}}(t)$.

With $x_{c}$ as given in equation (32), we find that the condition is likely met. Fig. 5 plots $\Delta_{\text {diff }} / \Delta_{\text {adv }}$ as a function of time for our simulation model Run-35-MHD-40-SNR and several values of $\eta \leq$ 10 and $v_{\text {sync } \leq 1 \mathrm{Ghz}}$. To be noted from the figure is that $\Delta_{\text {diff }} / \Delta_{\text {adv }} \ll$ 1 for times $\leq 200 \mathrm{kyr}$, and consequently we can ignore cosmic ray diffusion in the production of radio emission maps.

\subsubsection{Non-thermal emission maps}

We generate non-thermal radio emission maps from our MHD models of supernova remnants at representative points of time in the evolution of the supernova remnants. Using the procedure described in Section 2.4, we pre-compute for each viewing angle, $\theta_{\mathrm{obs}}$, the distribution of magnetic field normal to the line of sight, $B_{\perp}$. An illustrative example based on model Run-35-MHD-40 at time $80 \mathrm{kyr}$ is given in Fig. 6. Then, we compute the radio intensity with our modified version of the RADMC3D code. In Fig. 7, we show intensity maps for supernova remnant ages $6 \mathrm{kyr}$ (top), $20 \mathrm{kyr}$ (middle), and $80 \mathrm{kyr}$ (bottom) for a progenitor moving with $v_{\star}=20 \mathrm{~km} \mathrm{~s}^{-1}$. We selected three viewing angles to the equatorial plane, $\theta_{\mathrm{obs}}=0^{\circ}$ (left), $\theta_{\mathrm{obs}}=45^{\circ}$ (middle), and $\theta_{\mathrm{obs}}=90^{\circ}$ (right) and normalized the background-substracted maps. Fig. 8 displays corresponding radio maps for $v_{\star}=40 \mathrm{~km} \mathrm{~s}^{-1}$.

Model Run-35-MHD-20 with progenitor moving at $v_{\star}=$ $20 \mathrm{~km} \mathrm{~s}^{-1}$ traces the expanding supernova blastwave that is distorbed by its interaction with the circumstellar medium. Later, $40 \mathrm{kyr}$ after the explosion, the expanding shock wave has reached the unperturbed ISM and is fairly bright there. The radio arcs are now larger than at time $20 \mathrm{kyr}$ and the brightest region on the sides have a bilateral morphology. Note that the maps are background-subtracted, and the region of shocked stellar wind may be dimmer than the galactic radio background, leaving only the filamentary arcs prominently visible. For an inclination angle $\theta_{\mathrm{obs}}=45^{\circ}$ the remnants look rounder and more bubbly. One and the same remnant can appear with bilateral or arced structures depending on $\theta_{\mathrm{obs}}$. For $\theta_{\mathrm{obs}}=90^{\circ}$ the observer's 


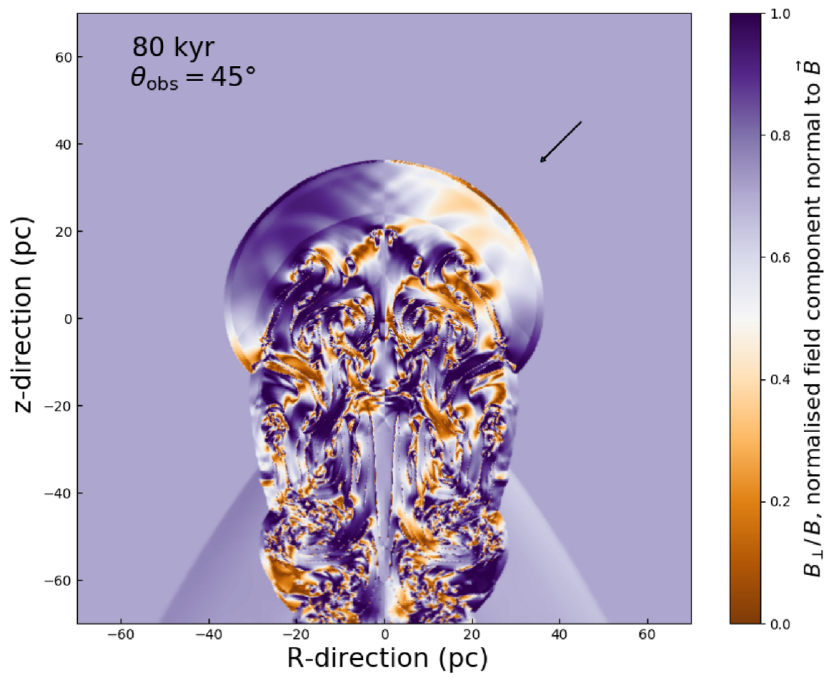

Figure 6. Normalized projection of the magnetic field perpendicular to the line of sight, $B_{\perp} / B$, for model Run-35-MHD-40 model with progenitor speed $v_{\star}=40 \mathrm{~km} \mathrm{~s}^{-1}$ at time $80 \mathrm{kyr}$ after the supernova explosion. The black arrow marks the direction of the observer's line of sight, making an angle $\theta_{\mathrm{obs}}=$ $45^{\circ}$ with the $z$-axis.

line of sight is aligned with the direction of stellar motion, and the projected remnant appears as a ring-like structure in the sky, on account of the two-dimensional nature of the simulations. Since the magnetic field is parallel to the stellar motion, $B_{\perp} \simeq 0$ in the shocked ISM, and the brightest emission originates from the regions of mixing, primarily in the reflected shock wave.

The radio intensity maps of our simulation Run-35-MHD-40 with progenitor speed $v_{\star}=40 \mathrm{~km} \mathrm{~s}^{-1}$ are shown in Fig. 8. The emission is brighter than in model Run-35-MHD-20 as a result of the faster progenitor star producing stronger shocks in its supernova remnant. At time $6 \mathrm{kyr}$ after the explosion, the shock wave has already been greatly distorted by the Wolf-Rayet circumstellar material and has lost sphericity to become as ovoid-like structure. Later in time, the shock wave adopts a hour-glass-like shape that appears spherical in the radio map for $\theta_{\mathrm{obs}}=45^{\circ}$ and to a lesser degree at $\theta_{\mathrm{obs}}=0^{\circ}$. After $40 \mathrm{kyr}$, the remnant has a bulb-like morphology that arises from both the shock wave expansion into the ISM and the channeling of the shock wave into the low-density cavity of unshocked stellar wind in the tail. The radio intensity peak shifts to the location where the shock wave intercepts the trail of stellar wind (Fig. 8d,e). The density in the region of wind-ISM interaction is more important than in the case of a progenitor moving with $v_{\star}=20 \mathrm{~km} \mathrm{~s}^{-1}$, and so the stabilizing effect of the magnetic field inside of the remnant is reduced. Hence, more ring-like structures appear in the emission maps, for example in Fig. 8(h). We would observe a series of concentric rings for $\theta_{\mathrm{obs}}=$ $90^{\circ}$, each of them corresponding to a ring in the trail of shocked stellar wind interacting with the channelled supernova shock wave. There is more variety and complexity in the radio appearance for fast-moving progenitors.

\section{DISCUSSION}

This section discusses the limitations of our method, compares our results to earlier results, and further examines our findings in the context of particle acceleration. Finally, we compare our results with observational data.

\subsection{Model limitations}

As any numerical study, our method suffers from simplifications that limit the realism of our results. The most obvious one is the cylindrical coordinate system with rotational invariance, which intrinsically imposes a symmetry axis to the models. This approach is convenient in the modelling of the circumstellar medium of massive stars and their subsequent supernova remnants (Franco et al. 1991; Rozyczka \& Tenorio-Tagle 1995; Comeròn 1997; Comerón \& Kaper 1998; van Marle, Langer \& García-Segura 2005, 2007; Ferreira \& de Jager 2008; van Marle et al. 2014; Green et al. 2019), at the expense of forcing an directional alignment of the motion of the progenitor, the local ISM magnetic field, and progenitor's axis of rotation. Only fully three-dimensional simulations permit flexibility in the directional arrangement (e.g. Katushkina et al. 2017, 2018), but they are far too expensive to permit scanning the parameter space of remnants from massive progenitors.

Supernova remnants from massive progenitors are multiphase regions composed of a warm, magnetized ISM through which the progenitor star moves, the evolving stellar wind, and a hot component produced by the interaction between the supernova shock wave and the material of the wind bubble and the shocked ISM gas. Supernova remnants may be located close to dense, cold molecular clouds that can further affect their evolution and modify the gas chemistry. The pressure of the cosmic rays accelerated in the supernova remnant (Ferrand, Decourchelle \& Safi-Harb 2014), anisotropic heat transfer (Orlando et al. 2005), photoionizing progenitor radiation, or the turbulence in the ISM should also be included in the models (Moranchel-Basurto et al. 2017; Villagran et al. 2020), but that is far beyond the scope of the current study and may be considered in future work. Last, note that intrinsic dense molecular (Zhou \& Chen 2011; Zhou et al. 2014, 2016) or low-density components (Arias et al. 2019a,b) of the ambient medium are an additional, in some context an even preponderant element to take into account in the shaping of core-collapse supernova remnants.

\subsection{Comparison with previous works}

This study extends earlier work beginning with Meyer et al. (2015) on hydrodynamical models of supernova remnants of runaway stars with initial 10,20 , and $40 \mathrm{M}_{\odot}$, which end their lives as red supergiants and generate Cygnus-Loop-like nebulae. The second study of this series explored the appearance of wind nebulae and remnants of a $60 \mathrm{M}_{\odot}$ progenitor star going through luminous-bluevariable and Wolf-Rayet phases, with emphasis on the mixing of material inside the remnant Meyer et al. (2020). What is new and different in the present study is the inclusion of the ISM magnetic field during both the pre- and the post-supernova phase, together with the post-processing of radio synchrotron intensity maps. The effect of ISM magnetization on the environment of massive stars has been investigated by van Marle et al. (2015), albeit without distinguishing ejecta from wind and ISM gas as we do by means of passive scalar tracers. Moreover, our models include a state-of-art stellar evolutionary model for the wind history of the $35 \mathrm{M}_{\odot}$ star that we concentrate on.

\subsection{Comparison with observations}

\subsubsection{Remnants of core-collapse supernovae}

Katsuda et al. (2018) determined several properties, such as the distance and the progenitor mass, of core-collapse supernova 


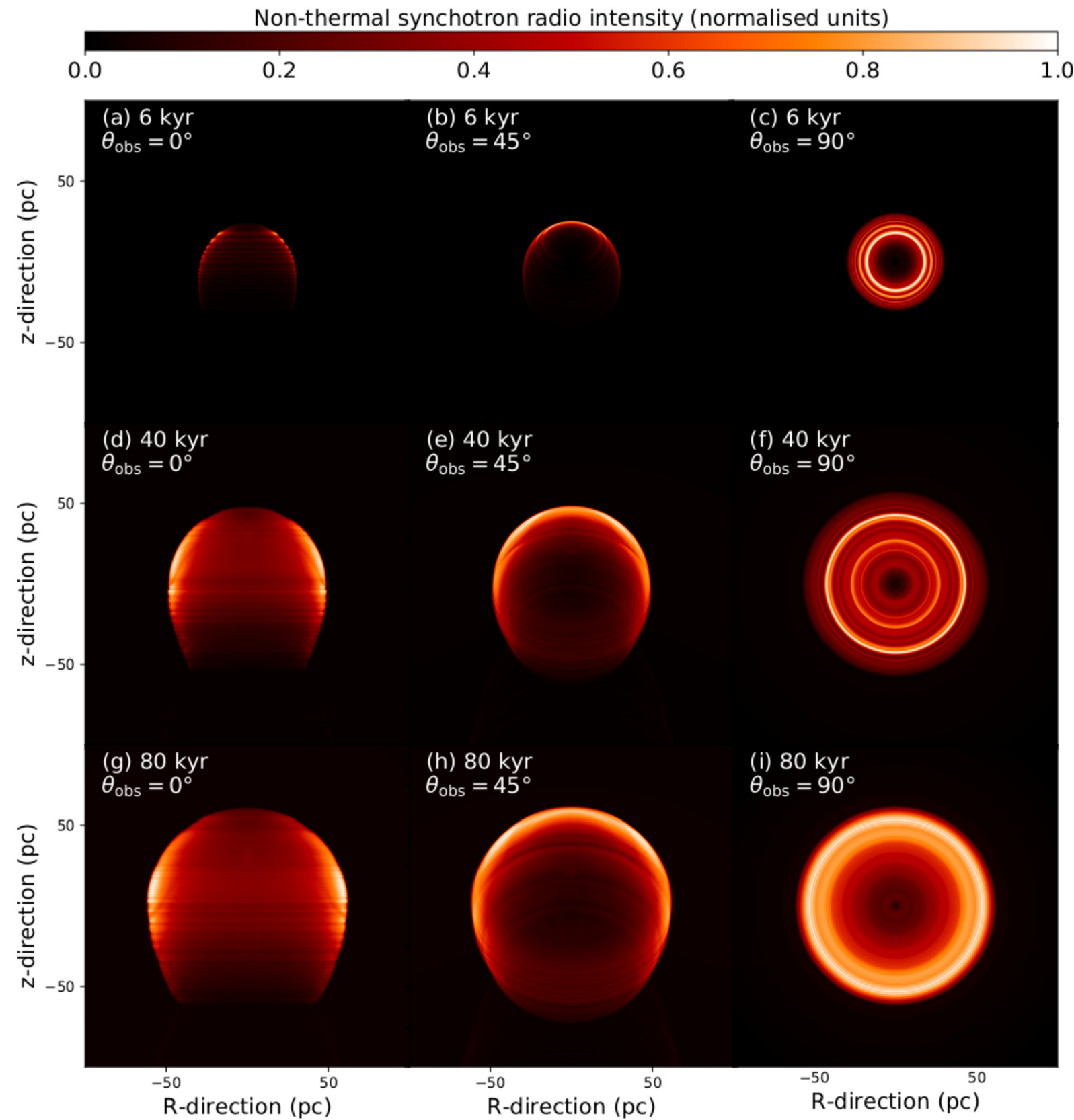

Figure 7. Normalized maps of radio synchrotron intensity of supernova remnants with progenitor speed $v_{\star}=20 \mathrm{~km} \mathrm{~s}^{-1}$ at times $6 \mathrm{kyr}$ (top), $40 \mathrm{kyr}$ (middle), and $80 \mathrm{kyr}$ (bottom) after the supernova explosion, respectively. The viewing angle between the equatorial plane and the line of sight is $\theta_{\mathrm{obs}}=0^{\circ}$ (left), $\theta_{\mathrm{obs}}=$ $45^{\circ}$ (middle), and $\theta_{\mathrm{obs}}=90^{\circ}$ (right).

remnants in the Milky Way and in low-metallicity dwarf galaxies such as the Large and Small Magellanic Clouds. They found that most of the identified remnants of massive progenitors in the Galaxy have a zero-age main-sequence mass $\geq 22.5 \mathrm{M}_{\odot}$. There is a general agreement between predictive stellar evolution models that the progenitor exploded with such mass, either as a red supergiant or as a Wolf-Rayet star, although more exotic situations such as blue supergiant progenitor star exist (Hillebrandt et al. 1987). Note that, in the context of massive binary systems, the explosion of the component can kick the companion, producing runaway stars (Lux et al. 2021). Our models explore the WolfRayet possibility using a zero-age main-sequence $35 \mathrm{M}_{\odot}$ star. As we concentrate on the evolution of rather older remnants, about
6-80 kyr after the explosion, our predictions are applicable to the objects listed between Kes 79 and W51C in table 1 of Katsuda et al. (2018).

Our models constitute baseline models to be further tailored to specific supernova remnants, in particular Kes 79, G350.10.3, G292.0+1.8, RX J1713.7-3946, Kes 79, G290.1-0.8, 3C 391, W44, G284.3-1.8, or CTB109. Note that C-, N-, and O-enriched material, witness of post-main-sequence winds from massive stars, has been directly observed in the remnant G296.1-0.5 (Castro et al. 2011), making it an evident candidate of a supernova remnant with Wolf-Rayet progenitor that is worth exploring numerically with simulations like ours. As underlined by Katsuda et al. (2018), the distribution of core-collapse supernovae in the Galaxy does not fit 
Non-thermal synchotron radio intensity (normalised units)

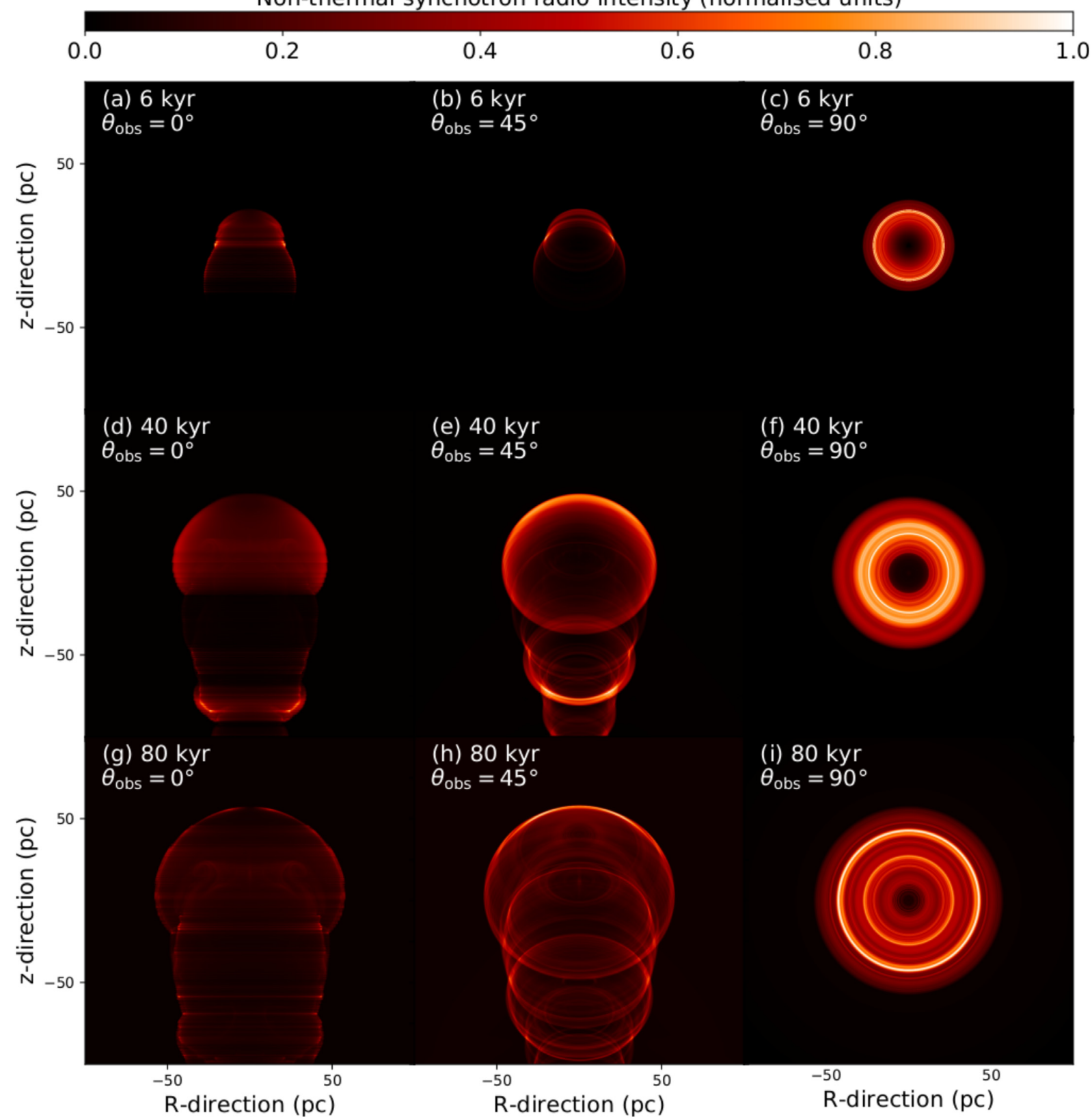

Figure 8. As Fig. 7 but here for model Run-35-MHD-40-SNR with progenitor moving with $40 \mathrm{~km} \mathrm{~s}^{-1}$.

any initial mass function, which suggest that there should be many more unidentified remnants our simulations would be applicable to. Note also that models for core-collapse remnants do not generally apply to the so-called historical supernova remnants since these are mostly of Type Ia (Green \& Stephenson 2003), except for Cas A (van Veelen et al. 2009; Zhou et al. 2018) and RWC 86 (Gvaramadze et al. 2017), respectively.

The space motion of the progenitor is the other fundamental ingredient of our simulations, together with the zero-age main-sequence mass. If the massive progenitor is at rest, then its circumstellar wind bubble remains spherical (Weaver et al. 1977). The star and the centre of its subsequent explosion are located at its centre (Freyer et al. 2006; Dwarkadas 2007). A sub-sonic motion of the progenitor will off-centre the remnant with respect to the wind bubble without changing its overall appearance (Meyer et al. 2020). Hence, remnants from slowly moving progenitor star should reflect the spherical symmetry of their circumstellar medium. However, as in situ star formation does not seem to be an obvious route to explain isolated Wolf-Rayet stars (Gvaramadze et al. 2012; Meyer et al. 2020), static massive stars should live and die inside their parent star formation region, where they would participate in the regulation of subsequent star formation (Paron et al. 2009). The feedback from stellar winds and/or jets of other (young) stellar objects (Bally, Reipurth \& Davis 2007; Fendt 2009; Fendt \& Sheikhnezami 2013) will affect the medium in which massive stars form (Murray, Goyal \& Chang 2018), evolve, and die. This should result in a very complex morphology, possibly further complicated by ISM cavities and enhanced levels of turbulence, in the ambient medium hosting a huge mix of material in a super bubble in which the supernova subsequently explodes (van Marle et al. 2012). 


\subsubsection{Comparison with specific objects}

Most supernova remnants in the Milky Way lie within $5^{\circ}$ of the galactic plane. The dilute ISM at high galactic latitudes makes (circumstellar) shocks weaker, resulting in fainter supernova remnants such as, for example, the rather evolved radio source G181.1+9.5 (Kothes et al. 2017). This latitude-dependence of the radio surface brightness of supernova remnants is known as the $\Sigma-\mathrm{D}$ relation (Caswell \& Lerche 1979). The modelled $35 \mathrm{M}_{\odot}$ runaway star moving with $v_{\star}=40 \mathrm{~km} \mathrm{~s}^{-1}$ travels about $220 \mathrm{pc}$ before exploding, about the same as the height of G181.1+9.5 above the galactic plane $(\approx 250 \mathrm{pc})$. Dedicated simulations would be highly desirable to explore the differences in the radio properties between supernova remnants of runaway progenitors in the Galactic place and those at higher galactic latitudes, as well as the effects of metallicities. Our non-thermal radio emission maps authorize a couple of further, direct comparisons with supernova remnants of massive, evolved progenitors, namely G296.5+10.0, the shell-type remnants CTB 109, and Kes 17.

First, G296.5+10.0 is a supernova remnant of core-collapse origin, confirmed by the trace of magnetized wind in which the supernova shock wave expands, and the presence of a neutron star therein (Harvey-Smith et al. 2010). Radio observations with the Australia Telescope Compact Arrary at $1.4 \mathrm{GHz}$ reveal a bipolar shape, which qualitatively similar to those in our model with velocity $v_{\star}=20 \mathrm{~km} \mathrm{~s}^{-1}$, Run-35-MHD-20-SNR, at time $6 \mathrm{kyr}$, when the shock wave interacts with the stellar-wind bow shock and accelerates electrons (Fig. 7a). Similarly, Fig. 8(a) also resembles greatly the 7 kyr old bilateral supernova remnant G296.5+10.0, implying that its progenitor might have been a rather fast-moving star of mass 20-40 $\mathrm{M}_{\odot}$. Secondly, the shell-type remnant CTB 109 is the remnant of a core-collapse supernova remnant of a $30-40 \mathrm{M}_{\odot}$ progenitor, which matches the mass range of the $35-\mathrm{M}_{\odot}$ stellar model used in this study. Its age is around $14000 \mathrm{yr}$, see Katsuda et al. (2018).

According to our results, CTB 109 should be surrounded by a circumstellar structure, i.e. a red-supergiant wind bubble engulfing a Wolf-Rayet ring, with which the supernova shock wave interacts, although its overall shape has been reproduced in the context of a type Ia explosion, i.e. without the presence of a dense circumstellar wind bubble generated by a massive progenitor (Bolte, Sasaki \& Breitschwerdt 2015). Its opened shell appearance, e.g. as seen with the Canadian Galactic Plane Survey at $1420 \mathrm{MHz}$ (Kothes \& Foster 2012), is similar to our model Run-35-MHD-SNR at times 6-40 kyr (Fig. 8b,e). Note also that CTB 109 is a hadronic gamma-ray emitter (Castro et al. 2012). The last example is the supernova remnant Kes 17 that is less than $40000 \mathrm{yr}$ old and with a progenitor mass 25$30 \mathrm{M}_{\odot}$ consistent with the Wolf-Rayet scenario (Katsuda et al. 2018). Its double-arced morphology observed with the Australian Telescope Array at $20 \mathrm{~cm}$ resembles our model Run-35-MHD-40-SNR at times 40 kyr (Fig. 8c).

\section{CONCLUSIONS}

We explore the formation, structure, and radio signatures of supernova remnants of massive, Wolf-Rayet-evolving supernova progenitors ejected from their parent cluster and moving through the ISM of the Milky Way. Our study concentrates on the coupled impact of stellar motion and the magnetization of the ISM. We perform MHD simulations over the entire stellar lifetime, as they successively evolve through a long main-sequence phase, a red supergiant, and a Wolf-Rayet phase, and eventually spawn a corecollapse supernova remnant. Numerical models are performed with the PLUTO code (Mignone et al. 2007, 2012) by simulating the circumstellar medium of massive stars, into which we launch a core-collapse supernova shock wave. We follow its interaction with the stellar surroundings and the local ambient ISM supported by an organized $7 \mu \mathrm{G}$ magnetic field. Considering two speeds of the runaway progenitors and running the simulations up to the oldest evolutionary phase of the supernova remnants, $150 \mathrm{kyr}$ after the explosion, their morphologies, their structures, the mixing of material happening in them are explored.

The presence of an ISM magnetic field profoundly affects the gas properties. Prior to the supernova explosion, the compressed magnetic field in the circumstellar medium stabilizes the wind/ISM contact discontinuity in the tail of the bubble. Indeed, compressed magnetic field in the outer remnant stabilizes and elongates the wind/ISM contact discontinuity of the cavity of unshocked stellar wind, in which the ejecta is channelled. A consequence is a reduced mixing efficiency of ejecta and evolved stellar-wind material enriched in $\mathrm{C}, \mathrm{N}$, and $\mathrm{O}$ elements in the inner region of the remnant, where the supernova shock wave propagates. Moreover, after the supernova explosion, the density downstream of the supernova shock front is reduced in our MHD simulations when it propagates into the pristine ambient medium, on account of the damping of turbulence. This must influence the acceleration processes of cosmic ray electrons and protons in supernova remnants from massive progenitors and will be investigated in future works (Bhatt et al. 2020). We emphasize the need for a careful treatment of the gas microphysics to properly simulate young supernova remnants interacting with circumstellar structures. This particularly applies to runaway massive progenitors whose supernova shock front is reverberated towards the centre of the explosion, generating a complex region made of shocks, discontinuities, and filamentary structures, in which nonthermal particles can be accelerated.

Last, using our modified version of the radiative transfer code RADMC/3D (Dullemond 2012) we produced synthetic radio-intensity maps showing projected arcs and filaments that we interpret as a morphological characteristic of supernova remnants of fast-moving Wolf-Rayet stars. Our radio predictions are qualitatively in accordance with the morphology of several core-collapse remnants, such as the bilateral G296.5+10.0, as well as the shell-type supernova remnants CTB 109 and Kes 17, identified as originating from 25$40 \mathrm{M}_{\odot}$ progenitors (Katsuda et al. 2018) that might have undergone a Wolf-Rayet phase. Our simulations and predictions regarding the non-thermal emission of supernova remnants from massive progenitors are relevant for and may be applied to the various galactic and extragalactic core-collapse remnants (Katsuda et al. 2018).

\section{ACKNOWLEDGEMENTS}

Authors are grateful to the referee, P. Velazquez, for comments on synchrotron emission that greatly improved the quality of the manuscript. The authors thank Allard Jan van Marle from Ulsan National Institute of Science and Technology for his kind advices on MHD simulations of the surroundings of massive stars. The authors acknowledge the North-German Supercomputing Alliance (HLRN) for providing HPC resources that have contributed to the research results reported in this paper. MP acknowledges the Max Planck Computing and Data Facility (MPCDF) for providing data storage resources and HPC resources that contributed to test and optimized the PLUTO code. LMO acknowledges partial support by the Russian Government Program of Competitive Growth of Kazan Federal University. 


\section{DATA AVAILABILITY}

This research used the PLUTO code developed at the University of Torino by A. Mignone (http://plutocode.ph.unito.it/) and of the RADMC-3D code developed at the University of Heidelberg by C. Dullemond (https://www.ita.uni-heidelberg.de/ dullemond/ software/radmc-3d/). The figures have been produced using the Matplotlib plotting library for the Python programming language (https://matplotlib.org/). The data underlying this article will be shared on reasonable request to the corresponding author.

\section{REFERENCES}

Arias M., Domček V., Zhou P., Vink J., 2019a, A\&A, 627, A75

Arias M. et al., 2019b, A\&A, 622, A6

Aschenbach B., Leahy D. A., 1999, A\&A, 341, 602

Ávila-Aroche A., Velázquez P. F., Camps-Fariña A., Toledo-Roy J. C., Esquivel A., 2020, MNRAS, 495, 2112

Bally J., Reipurth B., Davis C. J., 2007, in Reipurth B., Jewitt D., Keil K., eds, Protostars and Planets V, Observations of Jets and Outflows from Young Stars, University of Arizona Press, Tucson. p. 215

Balsara D. S., Tilley D. A., Howk J. C., 2008, MNRAS, 386, 627

Baranov V. B., Krasnobaev K. V., Kulikovskii A. G., 1971, Sov. Phys. Dokl., 15,791

Bestenlehner J. M. et al., 2014, A\&A, 570, A38

Bhatt M., Sushch I., Pohl M., Fedynitch A., Das S., Brose R., Plotko P., Meyer D. M.-A., 2020, Astropart. Phys., 123, 102490

Bolte J., Sasaki M., Breitschwerdt D., 2015, A\&A, 582, A47

Brighenti F., D'Ercole A., 1994, MNRAS, 270, 65

Brighenti F., D'Ercole A., 1995a, MNRAS, 277, 53

Brighenti F., D'Ercole A., 1995b, MNRAS, 273, 443

Broersen S., Chiotellis A., Vink J., Bamba A., 2014, MNRAS, 441, 3040

Brott I. et al., 2011, A\&A, 530, A115

Castro D., Slane P. O., Gaensler B. M., Hughes J. P., Patnaude D. J., 2011, ApJ, 734, 86

Castro D., Slane P., Ellison D. C., Patnaude D. J., 2012, ApJ, 756, 88

Caswell J. L., Lerche I., 1979, MNRAS, 187, 201

Chevalier R. A., 1982, ApJ, 258, 790

Chiotellis A., Schure K. M., Vink J., 2012, A\&A, 537, A139

Comeròn F., 1997, A\&A, 326, 1195

Comerón F., Kaper L., 1998, A\&A, 338, 273

Drury L., 1983a, Space Sci. Rev., 36, 57

Drury L. O., 1983b, Rep. Prog. Phys., 46, 973

Dullemond C. P., 2012, Astrophysics Source Code Library, record ascl:1202.015, RADMC-3D: A multi-purpose radiative transfer tool

Dwarkadas V. V., 2007, ApJ, 667, 226

Ekström S. et al., 2012, A\&A, 537, A146

Eldridge J. J., Genet F., Daigne F., Mochkovitch R., 2006, MNRAS, 367, 186

Fang J., Yu H., Zhang L., 2017, MNRAS, 464, 940

Fendt C., 2009, ApJ, 692, 346

Fendt C., Sheikhnezami S., 2013, ApJ, 774, 12

Ferrand G., Decourchelle A., Safi-Harb S., 2014, ApJ, 789, 49

Ferreira S. E. S., de Jager O. C., 2008, A\&A, 478, 17

Franco J., Tenorio-Tagle G., Bodenheimer P., Rozyczka M., 1991, PASP, 103,803

Freyer T., Hensler G., Yorke H. W., 2003, ApJ, 594, 888

Freyer T., Hensler G., Yorke H. W., 2006, ApJ, 638, 262

Garcia-Segura G., Mac Low M.-M., Langer N., 1996, A\&A, 305, 229

Green D. A., Stephenson F. R., 2003, in Weiler K., ed., Lecture Notes in Physics, Vol. 598 , Supernovae and Gamma-Ray Bursters of Historical Supernovae. Springer-Verlag, Berlin, p. 7

Green S., Mackey J., Haworth T. J., Gvaramadze V. V., Duffy P., 2019, A\&A, 625, A4

Gvaramadze V. V., Kniazev A. Y., Fabrika S., 2010a, MNRAS, 405, 1047

Gvaramadze V. V., Kniazev A. Y., Fabrika S., 2010b, MNRAS, 405, 1047

Gvaramadze V. V., Weidner C., Kroupa P., Pflamm-Altenburg J., 2012, MNRAS, 424, 3037
Gvaramadze V. V., Menten K. M., Kniazev A. Y., Langer N., Mackey J., Kraus A., Meyer D. M.-A., Kamiński T., 2014, MNRAS, 437, 843

Gvaramadze V. V. et al., 2017, Nat. Astron., 1, 0116

Hamann W. R., Gräfener G., Liermann A., 2006, A\&A, 457, 1015

Harvey-Smith L., Gaensler B. M., Kothes R., Townsend R., Heald G. H., Ng C. Y., Green A. J., 2010, ApJ, 712, 1157

Hillebrandt W., Hoeflich P., Weiss A., Truran J. W., 1987, Nature, 327, 597

Janka H.-T., 2012, Annu. Rev. Nucl. Part. Sci., 62, 407

Janka H.-T., Melson T., Summa A., 2016, Annu. Rev. Nucl. Part. Sci., 66, 341

Jun B.-I., Norman M. L., 1996, ApJ, 472, 245

Katsuda S., Takiwaki T., Tominaga N., Moriya T. J., Nakamura K., 2018, ApJ, 863, 127

Katushkina O. A., Alexashov D. B., Izmodenov V. V., Gvaramadze V. V., 2017, MNRAS, 465, 1573

Katushkina O. A., Alexashov D. B., Gvaramadze V. V., Izmodenov V. V., 2018, MNRAS, 473, 1576

Kothes R., Foster T., 2012, ApJ, 746, L4

Kothes R., Reich P., Foster T. J., Reich W., 2017, A\&A, 597, A116

Langer N., 2012, ARA\&A, 50, 107

Lux O., Neuhäuser R., Mugrauer M., Bischoff R., 2021, preprint (arXiv:2101.05771)

Mackey J., Mohamed S., Neilson H. R., Langer N., Meyer D. M.-A., 2012, ApJ, 751, L10

Meyer D. M.-A., Gvaramadze V. V., Langer N., Mackey J., Boumis P., Mohamed S., 2014, MNRAS, 439, L41

Meyer D. M.-A., Langer N., Mackey J., Velázquez P. F., Gusdorf A., 2015, MNRAS, 450, 3080

Meyer D. M.-A., van Marle A.-J., Kuiper R., Kley W., 2016, MNRAS, 459, 1146

Meyer D. M.-A., Mignone A., Kuiper R., Raga A. C., Kley W., 2017, MNRAS, 464, 3229

Meyer D. M.-A., Petrov M., Pohl M., 2020, MNRAS, 493, 3548

Meyer D. M.-A., Oskinova L. M., Pohl M., Petrov M., 2020, MNRAS, 496, 3906

Mignone A., Bodo G., Massaglia S., Matsakos T., Tesileanu O., Zanni C., Ferrari A., 2007, ApJS, 170, 228

Mignone A., Zanni C., Tzeferacos P., van Straalen B., Colella P., Bodo G., 2012, ApJS, 198, 7

Moffat A. F. J. et al., 1998, A\&A, 331, 949

Moranchel-Basurto A., Velázquez P. F., Giacani E., Toledo-Roy J. C., Schneiter E. M., De Colle F., Esquivel A., 2017, MNRAS, 472, 2117

Moranchel-Basurto A., Velázquez P. F., Ares de Parga G., Reynoso E. M., Schneiter E. M., Esquivel A., 2020, MNRAS, 494, 1531

Moriya T. J., Förster F., Yoon S.-C., Gräfener G., Blinnikov S. I., 2018, MNRAS, 476, 2840

Munoz M., Moffat A. F. J., Hill G. M., Shenar T., Richardson N. D., Pablo H., St-Louis N., Ramiaramanantsoa T., 2017, MNRAS, 467, 3105

Murray D., Goyal S., Chang P., 2018, MNRAS, 475, 1023

Orlando S., Peres G., Reale F., Bocchino F., Rosner R., Plewa T., Siegel A., 2005, A\&A, 444, 505

Orlando S., Bocchino F., Reale F., Peres G., Petruk O., 2007, A\&A, 470, 927

Orlando S., Bocchino F., Reale F., Peres G., Pagano P., 2008, ApJ, 678, 274

Orlando S., Bocchino F., Miceli M., Petruk O., Pumo M. L., 2012, ApJ, 749, 156

Paron S., Ortega M. E., Rubio M., Dubner G., 2009, A\&A, 498, 445

Reynolds S. P., 2008, ARA\&A, 46, 89

Rozyczka M., Tenorio-Tagle G., 1995, MNRAS, 274, 1157

Rozyczka M., Tenorio-Tagle G., Franco J., Bodenheimer P., 1993, MNRAS, 261,674

Sander A., Hamann W. R., Todt H., 2012, A\&A, 540, A144

Sanyal D., Langer N., Szécsi D., -C Yoon S., Grassitelli L., 2017, A\&A, 597, A71

Schneiter E. M., Velázquez P. F., Reynoso E. M., de Colle F., 2010, MNRAS, 408, 430

Schneiter E. M., Velázquez P. F., Reynoso E. M., Esquivel A., De Colle F., 2015, MNRAS, 449, 88 
Shu F. H., 1992, The Physics of Astrophysics. Volume II: Gas Dynamics, University Science Books, Mill Valley

Smartt S. J., 2009, ARA\&A, 47, 63

Stock D. J., Barlow M. J., 2010, MNRAS, 409, 1429

Telezhinsky I., Dwarkadas V. V., Pohl M., 2012, Astropart. Phys., 35, 300

Telezhinsky I., Dwarkadas V. V., Pohl M., 2013, A\&A, 552, A102

Toalá J. A. et al., 2018, ApJ, 869, L11

Toledo-Roy J. C., Esquivel A., Velázquez P. F., Reynoso E. M., 2014, MNRAS, 442, 229

Truelove J. K., McKee C. F., 1999, ApJS, 120, 299

van Marle A. J., Langer N., García-Segura G., 2005, A\&A, 444, 837

van Marle A. J., Langer N., García-Segura G., 2007, A\&A, 469, 941

van Marle A. J., Smith N., Owocki S. P., van Veelen B., 2010, MNRAS, 407, 2305

van Marle A. J., Meliani Z., Keppens R., Decin L., 2011, ApJ, 734, L26

van Marle A. J., Meliani Z., Marcowith A., 2012, A\&A, 541, L8

van Marle A. J., Decin L., Meliani Z., 2014, A\&A, 561, A152

van Marle A. J., Meliani Z., Marcowith A., 2015, A\&A, 584, A49

van Veelen B., Langer N., Vink J., García-Segura G., van Marle A. J., 2009, A\&A, 503, 495

Velázquez P. F., Vigh C. D., Reynoso E. M., Gómez D. O., Schneiter E. M., 2006, ApJ, 649, 779

Velázquez P. F. et al., 2017, MNRAS, 466, 4851

Viallet M., Baty H., 2007, A\&A, 473, 1

Villagran M. A., Velázquez P. F., Gómez D. O., Giacani E. B., 2020, MNRAS, 491, 2855

Weaver R., McCray R., Castor J., Shapiro P., Moore R., 1977, ApJ, 218, 377

Whalen D., van Veelen B., O'Shea B. W., Norman M. L., 2008, ApJ, 682, 49

Wilkin F. P., 1996, ApJ, 459, L31

Woosley S. E., Heger A., Weaver T. A., 2002, Rev. Mod. Phys., 74, 1015

Yoon S.-C., Langer N., 2005, A\&A, 443, 643

Zhou P., Chen Y., 2011, ApJ, 743, 4

Zhou P., Safi-Harb S., Chen Y., Zhang X., Jiang B., Ferrand G., 2014, ApJ, 791, 87

Zhou P., Chen Y., Safi-Harb S., Zhou X., Sun M., Zhang Z.-Y., Zhang G.-Y., 2016, ApJ, 831, 192

Zhou P., Li J.-T., Zhang Z.-Y., Vink J., Chen Y., Arias M., Patnaude D., Bregman J. N., 2018, ApJ, 865, 6

Zirakashvili V. N., Ptuskin V. S., 2018, Astropart. Phys., 98, 21

\section{APPENDIX A: EMISSION COEFFICIENTS FOR NON-THERMAL SYNCHROTRON EMISSION}

To the best of our knowledge, three main recipes are available for the emission coefficient of non-thermal radio synchrotron emission in the context of supernova remnants (Jun \& Norman 1996; Orlando et al. 2007; Ávila-Aroche et al. 2020). Considering the electron spectrum in the vicinity of the shocks,

$N(E)=K E^{-s}$,

where $E$ is the electron energy and $s=2$ the index and $K \propto n$. They read as,

$j_{\text {sync }}^{\text {Orlando }}(v) \propto K B_{\perp}^{(s+1) / 2} v^{-(s-1) / 2}$,

with $\theta_{\text {obs }}$ the viewing angle of the observer, $B_{\perp}$ the magnetic field component perpendicular to the line of sight and $v$ the emission frequency:

$j_{\text {sync }}^{\mathrm{Jun}}(v) \propto K^{2-s} p^{s-1} B_{\perp}^{(s+1) / 2} v^{-(s-1) / 2}$,

where $p$ is the gas thermal pressure, and

$j_{\text {sync }}^{\text {Avila }}(v) \propto K v^{\prime 2(s-1)} B_{\perp}^{(s+1) / 2} v^{-(s-1) / 2}$,

where $v^{\prime}$ is the gas velocity in the rest frame of the explosion, respectively. This diagnostics has been widely used in, e.g. the context of the core-collapse but also Type Ia progenitors such as the historical supernova remnants Tycho (Moranchel-Basurto et al. 2020) and SN 1006 (Schneiter et al. 2015; Velázquez et al. 2017).

We generate comparative normalized non-thermal radio emission maps from two selected models of supernova remnants. First, one with a progenitor star moving rather slowly with velocity $20 \mathrm{~km} \mathrm{~s}^{-1}$, and in which the thermal pressure compares with the ram and magnetic pressures (Fig. A1). Secondly, a model with a fast-moving progenitor is moving with velocity $40 \mathrm{~km} \mathrm{~s}^{-1}$ and in which the ISM magnetic pressure is dynamically unimportant (Fig. A2). Fig. A1 a reveals the bright radio synchrotron circumstellar medium of the progenitor, produced by wind-ISM interaction before the explosion of the massive star, while Fig. A1(b,c) do not. The recipe used in Fig. A1(a) clearly overestimates particle acceleration from the forward shock of the stellar wind bubble, which is much weaker than the forward shock of the expanding supernova blastwave. Hence, the emission coefficient in Orlando et al. (2007) is not the most suitable to our core-collapse remnant problem. The models calculated with the other emission coefficients do not permit to select an optimal one for our study (Fig. A1b,c). The emission coefficient in equation (A3) of Jun \& Norman (1996) has a dependence on the thermal pressure $p$, implying that it is sensitive to cooling and heating by optically thin radiative cooling processes and therefore traces the fast shocks well. Similarly, the recipe of emission coefficient in equation (A4) of Ávila-Aroche et al. (2020) goes as $j_{\text {sync }}^{\text {Avila }}(v) \propto v^{2}$, which imposes a strong dependence of the chosen frame in which we simulate the stellar wind bubble and the supernova explosion. Consequently, we decide in our study to use the recipe of Jun \& Norman (1996). 
Non-thermal synchotron radio intensity (normalised units)

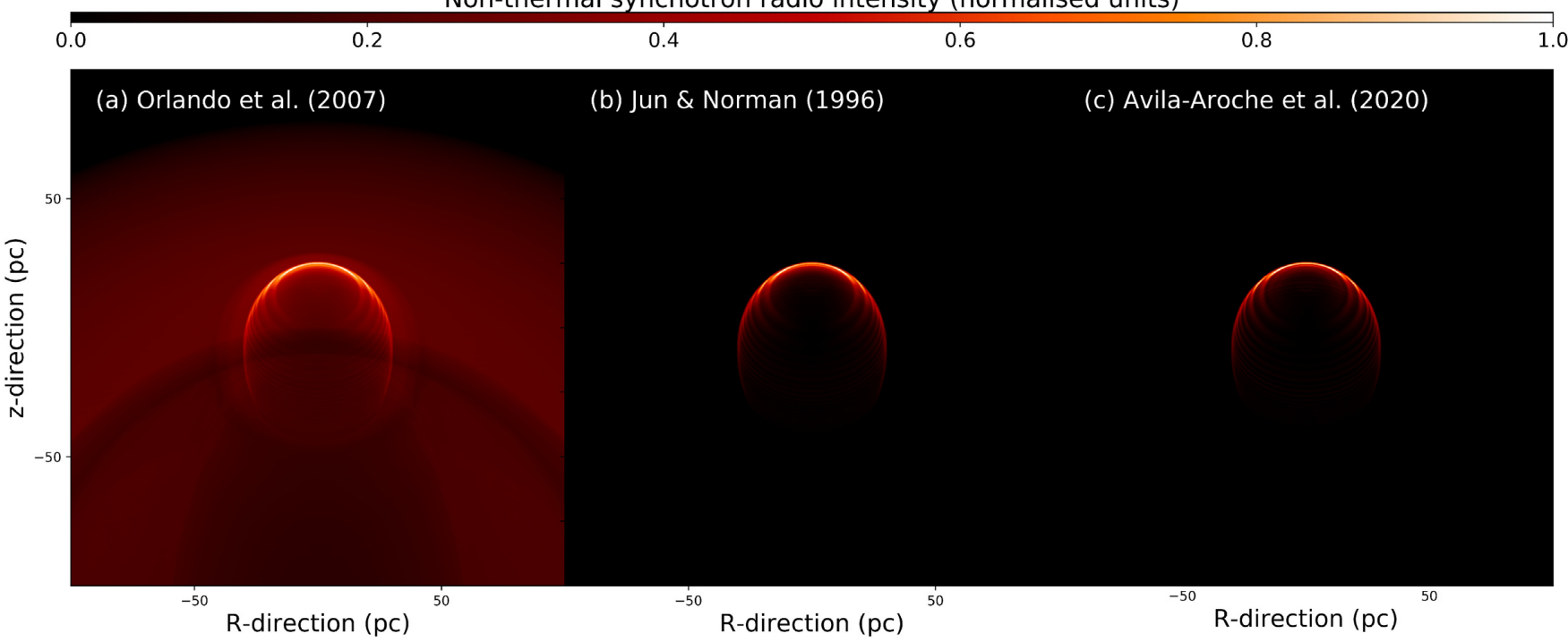

Figure A1. Normalized maps of radio synchrotron intensity of supernova remnants with progenitor speed $v_{\star}=20 \mathrm{~km} \mathrm{~s}^{-1}$ at time $6 \mathrm{kyr}$, and calculated using several prescriptions for the non-thermal emission coefficient. The viewing angle between the equatorial plane and the line of sight is $\theta_{\mathrm{obs}}=45^{\circ}$ (middle).

Non-thermal synchotron radio intensity (normalised units)

$\begin{array}{llllll}0.0 & 0.2 & 0.4 & 0.6 & 0.8 & 1.0\end{array}$

(a) Orlando et al. (2007)

(b) Jun \& Norman (1996)

(c) Avila-Aroche et al. (2020)

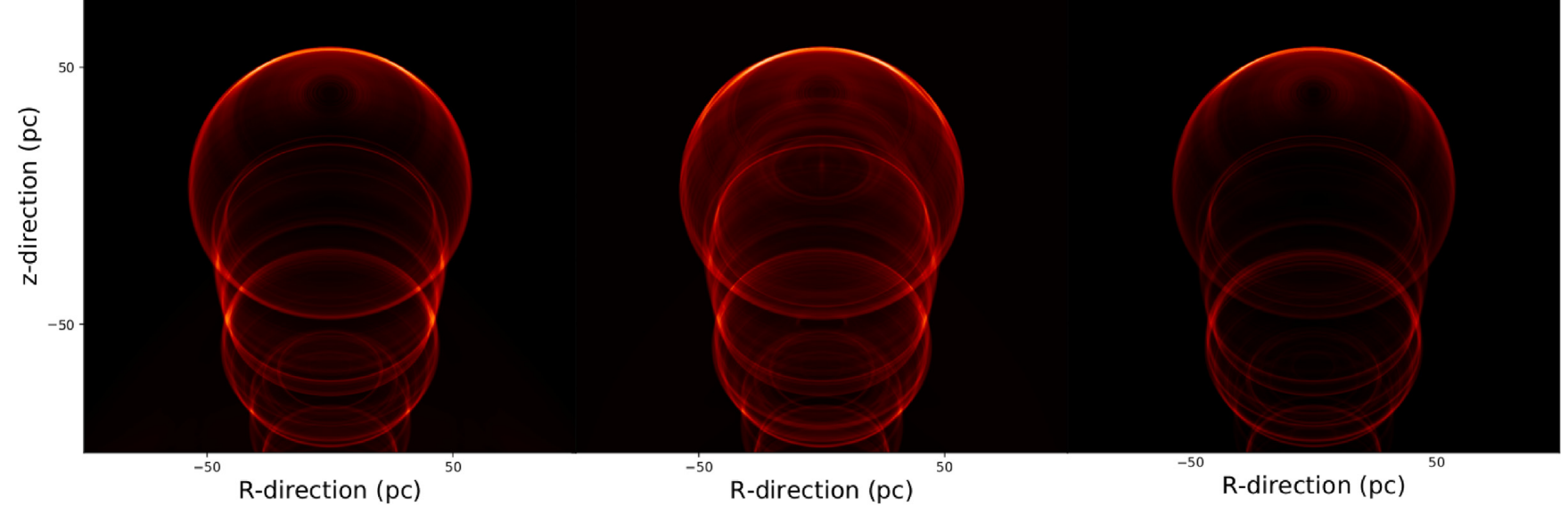

Figure A2. Same as Fig. A1 with progenitor speed $v_{\star}=40 \mathrm{~km} \mathrm{~s}^{-1}$ at time $80 \mathrm{kyr}$.

This paper has been typeset from a $\mathrm{T}_{\mathrm{E}} \mathrm{X} / \mathrm{LT} \mathrm{E} \mathrm{X}$ file prepared by the author. 